\title{
Congestion and Awareness Control in Cooperative Vehicular Systems
}

\author{
This paper surveys methods to ensure that each vehicle can detect and \\ communicate with neighboring vehicles in order to avoid saturation of the \\ V2X channel and prevent dangerous instability.
}

\author{
By Miguel Sepulcre, Jens Mittag, Paolo Santi, Member IeEe, \\ Hannes Hartenstein, Member IEeE, and Javier Gozalvez, Senior Member IEeE
}

ABSTRACT | Cooperative vehicular systems have been identified as a promising solution to overcome the current and future needs for increasing traffic safety and efficiency, while providing infotainment and added-value services on the move. To achieve their objectives, cooperative vehicular systems will be based on wireless communications between vehicles and with other infrastructure nodes, and will have to deal with highly dynamic nodes, challenging propagation conditions, and stringent application requirements. By looking at cooperative applications and their data traffic, as well as the current and foreseen spectrum allocations for cooperative vehicular systems, there is a risk that the corresponding radio channels could easily be saturated if no control algorithms are used. The saturation of the radio channels would result in unstable vehicular communications, and thus in an inefficient operation of cooperative systems. As a prime example of upcoming ubiquitous networks contributing to the vision of "a thousand radios per person," cooperative vehicular systems

Manuscript received June 17, 2010; revised October 6, 2010 and December 25, 2010; accepted February 1, 2011. Date of publication June 2, 2011; date of current version June 17, 2011. The work of M. Sepulcre and J. Gozalvez was supported by the FP7 ICT Project iTETRIS (FP7 224644), the Spanish Ministerio de Fomento (T39/2006), and the Generalitat Valenciana (BFPI06/126). The work of J. Mittag was supported by the Ministry of Science, Research and the Arts of Baden-Württemberg (AZ: Zu 33-827.377/19,20), the Klaus Tschira Stiftung, the INIT GmbH, the PTV AG for the research group on Traffic Telematics for the research group on Traffic Telematics. M. Sepulcre and J. Gozalvez are with the Uwicore Laboratory, University Miguel Hernandez of Elche, 03202 Elche, Spain (e-mail: msepulcre@umh.es; j.gozalvez@umh.es).

J. Mittag is with the Institute of Telematics, Karlsruhe Institute of Technology, 76131 Karlsruhe, Germany (e-mail: jens.mittag@kit.edu).

P. Santi is with the Istituto di Informatica e Telematica del CNR 56124 Pisa, Italy (e-mail: paolo.santi@iit.cnr.it).

H. Hartenstein is with the Steinbuch Centre of Computing, Karlsruhe Institute of Technology, 76131 Karlsruhe, Germany (e-mail: hartenstein@kit.edu).

Digital Object Identifier: 10.1109/JPROC.2011.2116751 need to be designed to scale to high densities of radios without centralized coordination, while at the same time guaranteeing the requirements of the implemented applications and services, for example the stringent needs of active traffic safety applications. In this paper, we survey and classify various decentralized methods to control the load on the radio channels and to ensure each vehicle's capacity to detect and communicate with the relevant neighboring vehicles, with a particular focus on approaches based on transmit power and rate control. Finally, we discuss the open research challenges that are imposed by different application requirements and potential existing contradictions.

KEYWORDS | Awareness control; congestion control; cooperative vehicular systems; power control

\section{INTRODUCTION}

Foreseen cooperative systems for intelligent transportation systems (ITS) address the current and future needs of increasing traffic safety, efficiency and comfort. Despite the predicted growth rates in the number of motorized vehicles and the volume of transported goods, transportation should become safer, cleaner, more efficient and more comfortable. To help to reach these goals, cooperative vehicular systems will enable the direct exchange of information between vehicles, and between vehicles and road side units (RSUs), using the IEEE 802.11p [1] technology on the $5.9 \mathrm{GHz}$ band. This technology is based on the carrier sense multiple access with collision avoidance (CSMA/CA) access protocol, and is being adapted to the European context in the ETSI ITS-G5 standard [2]. The operation of cooperative vehicular systems is currently 
based on the exchange of two primary types of messages. On the one hand, cooperative awareness messages (CAMs), also known as beacons, are broadcasted by all nodes on the so-called control channel, to provide and receive status information about the presence, geographical position and movement of neighboring nodes, and service announcements to/from those nodes. On the other hand, event-driven emergency messages are transmitted when an abnormal or dangerous situation is detected, in order to inform surrounding nodes about it.

As the technology becomes more widely adopted, and cooperative applications and services are deployed, the shared radio channels could easily be saturated. It is well known from wireless local area networks based on CSMA/CA, that communication performance might not degrade gracefully if the network is saturated, but will in fact drop significantly once the maximum capacity is exceeded. Since the exchange of periodic CAMs alone could already saturate the channel, methods are required to control and limit the load on the radio channel. Congestion control has been studied in depth in various areas of computer networking. The term congestion control typically goes together with the transport control protocol (TCP) of the Internet protocol suite. Here, cooperative control is used to ensure that each TCP connection gets a fair share of the available network resources. The mechanisms for congestion control in vehicular networks typically show analogous concepts, like decentralized control and fairness, but differ significantly due to the specific constraints of wireless communications in highly mobile and potentially harsh radio conditions.

In addition to guaranteeing a channel load level that ensures stable system operation, cooperative vehicular systems will be required to ensure connectivity among the vehicular nodes imposed by the implemented applications. To ensure network-wide connectivity through the dynamic adaptation of each node's transmission parameters, topology control protocols have been proposed for wireless ad-hoc and sensor networks [3]. However, the presence of highly dynamic vehicular mobility, along with the harsh radio propagation conditions strongly challenge the establishment of stable vehicular connections, and thus reduce the feasibility of traditional topology control protocols. In addition, cooperative vehicular systems do not require network-wide connectivity and the establishment of links in the traditional sense, but rather accurate and updated data on each vehicle's local environment (e.g., position, speed and direction of movement of neighboring vehicles) to support upper-layer protocols and cooperative applications. In this context, this paper defines awareness control protocols as those techniques aimed at ensuring each vehicle's capacity to detect, and possibly communicate with the relevant vehicles and infrastructure nodes present in their local neighborhood, through the dynamic adaptation of their transmission parameters. Awareness control protocols can, for example, adapt each vehicle's

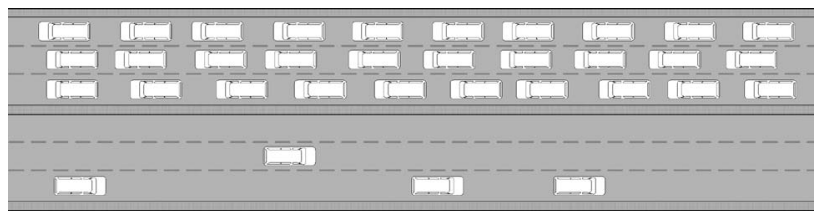

Fig. 1. Highway scenario with a traffic jam in one direction of driving and free flow conditions in the other direction. This example represents a typical traffic situation in which congestion control and awareness control protocols might be needed.

transmission power to successfully transmit a message at a given distance, or dynamically modify each vehicle's packet generation rate to increase the probability of receiving at least one packet at a certain distance during a given time window. Given their similarities, awareness control can be seen as a geolocalized adaptation of topology control.

Based on the previous definitions and their fundamentally different objectives, congestion and awareness control can be easily differentiated. For instance, congestion control aims to limit the observed load on the wireless channel for all nodes in order to provide fair and harmonized access to the wireless medium. As such, congestion control algorithms reduce the transmission power or rate of all nodes in order to avoid scenarios in which neighboring nodes, which are part of the same traffic situation, use (on average) significantly different power levels or beaconing rates. Considering the example illustrated in Fig. 1, the high density of vehicles in the traffic-congested area would require the use of congestion control protocols to control and limit the channel load. Unlike congestion control protocols, awareness control algorithms adjust the power or rate of only a selected subset of nodes, with the objective of fulfilling the requirements of a particular application. In the scenario illustrated in Fig. 1, the requirements of the applications run by the vehicles in the traffic jam are notably different from those of the applications run by the vehicles under free-flow conditions moving in the opposite direction, with different speeds and distances between neighbouring vehicles. For example, while vehicles under free-flow conditions would require their communication settings to allow for a safe lanechange maneuver, such a maneuver could be completely unexpected, or be less dangerous, for vehicles in the traffic jam. Awareness control protocols would be therefore required to dynamically adapt each vehicle's communications parameters to efficiently satisfy their individual requirements.

In this context, this paper focuses on congestion and awareness-control techniques with a special emphasis on transmit power control and application-driven design policies. Recently, various researchers contributed approaches and performance evaluations that address the issues of controlling the load on the radio channel, and 
guaranteeing each vehicle's capacity to communicate with its local neighborhood. In this paper, we survey various key approaches and key findings in a coherent manner to present and categorize the pool of ideas for upcoming standardization and deployment activities. We will discuss in some detail three approaches based on transmit power control, one targeting the issue of congestion control, and two addressing an efficient awareness control. The paper is structured accordingly. We first introduce some background information in Section II, followed by a discussion of congestion and awareness control through the perspective of control theory in Section III. In Section IV, we survey congestion control approaches that have been proposed for vehicular communications, and discuss in detail a proposal based on transmit power control, in addition to evaluating its performance vs. effectiveness tradeoffs. Section V surveys key contributions to awareness control, before discussing geo-opportunistic and traffic contextual approaches designed to ensure the strict requirements imposed by cooperative applications, in particular traffic safety applications. Section VI discusses open research challenges deriving from the joint study of congestion and awareness control protocols, as well as multi-application scenarios. Finally, Section VII summarizes the main contributions made by this paper.

\section{BACKGROUND}

A first generation of future cooperative vehicular systems will be based on the IEEE 802.11p standard, according to current standardization activities. As such, previous studies dealing with congestion and awareness control have been performed on top of IEEE 802.11p. In order to support the explanations and descriptions given in the following sections and to clarify the system setup and the assumptions made in this paper, we will briefly elaborate on the relevant aspects of the communication system for cooperative vehicular systems.

IEEE 802.11p [1] is specified to operate in the $5.9 \mathrm{GHz}$ frequency band. At the medium access control (MAC) layer, it employs the CSMA/CA mechanism to coordinate medium access by multiple stations. In CSMA/CA, each station has to listen to the channel and check whether it is free before being allowed to transmit. This operation is called carrier sensing, and it is performed by comparing the detected energy on the channel with a pre-defined threshold, called the carrier sensing threshold. The region of space where a certain ongoing transmission can be detected by a device tentatively accessing the channel is called the carrier sensing region. Note that, in general, this region has irregular shape due to nonisotropic radio signal propagation. However, it is a common practice in the wireless networking literature to consider the carrier sensing region as nearly circular, hence the notion of carrier sensing range used to refer to the distance up to which an ongoing transmission can be sensed by a device attempting to access the channel. If the channel is busy, the station has to defer, wait until the channel is free again and choose a random backoff timer that determines the additional waiting time that has to elapse after the channel is sensed idle. Despite the backoff mechanism, two or more stations can transmit simultaneously, therefore producing a packet collision and a possible data loss due to interferences. In particular, two stations can transmit simultaneously mainly due to the well known hiddenterminal problem. The hidden-terminal problem occurs when two (or more) stations cannot detect each other's transmissions, but their transmission ranges are not disjoint. It has been widely studied in the literature.

Due to its robustness against fast fading channels, the IEEE 802.11p amendment adapts the orthogonal frequency division multiplexing (OFDM) transmission technology used in IEEE 802.11a and g, with the exception that $10 \mathrm{MHz}$ instead of $20 \mathrm{MHz}$ channels are used by default. The reduction from 20 to $10 \mathrm{MHz}$ was necessary to account for the increased Doppler and delay spreads (as reported by [4] or [5]) which would otherwise lead to inter-symbol interference (ISI) and inter-carrier interference (ICI), and thus significantly challenge the successful reception of packets. Apart from the problem of ISI and ICI, a receiver is also challenged by the fast fading channel conditions that are observable due to the high relative mobility of vehicles. For instance, the coherence time of the channel, i.e. the time during which the channel impulse response is essentially invariant, can be smaller than the duration of a single packet transmission [6], which could result in an increased probability of bit and packet errors. This is an issue, since the IEEE 802.11p frame format provides only a preamble to fully estimate the channel and only four pilot subcarriers to partially track the state of the channel [7]. Hence, the initial estimate can become invalid at the end of the reception leading to an increased probability of bit and packet errors [6]. As a consequence, the channel impulse response of two consecutive packet transmissions will most likely not be correlated, and the channel can only be considered symmetric for an instant of time, but not for slightly different timestamps. By symmetric, we mean that the propagation characteristics of the radio channel are approximately the same in both directions of the wireless communication link.

As defined by the Federal Communications Commission of the USA (FCC) [8], a spectrum of $75 \mathrm{MHz}$ has been allocated at $5.9 \mathrm{GHz}$. Similarly, a spectrum of $50 \mathrm{MHz}$ has been allocated at the same frequency band in Europe. In both cases, the entire spectrum is divided into several $10 \mathrm{MHz}$ channels, out of which one channel, commonly called the Control Channel $(\mathrm{CCH})$, is used as a reference for the exchange of safety-related information. The remaining channels are known as service channels and are used for safety and non-safety applications. The data rates provided by IEEE 802.11p [1] with such $10 \mathrm{MHz}$ channels range from 3 to $27 \mathrm{Mb} / \mathrm{s}$. While the lower data rates are the 
Table 1 The Capacity of Multilane Highways and the Corresponding Average Speeds According to [10]. In Addition, the Number of Vehicles Within the Communication Range are Listed for a 3 Lane per Direction Highway and a 1000 m Communication Range

\begin{tabular}{|cccc|}
\hline $\begin{array}{c}\text { Level } \\
\text { of Service }\end{array}$ & $\begin{array}{c}\text { Max. density } \\
\text { (vehicles/km/lane) }\end{array}$ & $\begin{array}{c}\text { Avg. Speed } \\
(\mathrm{km} / \mathrm{h})\end{array}$ & $\begin{array}{c}\text { Vehicles within } \\
\text { 1000 m comm. range }\end{array}$ \\
\hline A & 7 & 100.0 & 84 \\
B & 11 & 100.0 & 132 \\
C & 16 & 98.4 & 192 \\
D & 22 & 91.5 & 264 \\
E & 25 & 88.0 & 300 \\
\hline
\end{tabular}

most robust ones and require lower signal-to-interference and noise ratio to correctly decode a packet, higher data rates come with the benefit of reduced transmission times, and thus with the possible gain of a reduced packet collision probability in situations of higher channel loads. Obviously, there is tradeoff between increased robustness and reduced congestion. To investigate this tradeoff, a simulation study was performed in [9] in order to determine the most robust data rate for broadcast communication. The study's results reveal that a $6 \mathrm{Mb} / \mathrm{s}$ data rate turns out to be the best selection for safety related communication. Since recent standardization activities [2] have taken up these findings, we will assume a fixed data rate of $6 \mathrm{Mb} / \mathrm{s}$ in the rest of this paper.

Apart from the key technology characteristics, it is also important to understand how it is envisioned that the cooperative vehicular system will operate and what its dimensions will be. According to the common agreement among researchers and industry, vehicles will periodically broadcast CAMs in order to establish a mutual awareness. CAM messages provide information on positioning, speed, and heading, among other fields. The establishment of a mutual awareness can be considered the fundamental safety service in cooperative vehicular systems, on top of which advanced safety applications, e.g., cooperative forward collision warning or intersection collision warning, will be deployed. However, in order to fulfill the requirements of such advanced safety applications, the transmitted information might need to be updated several times per second, possibly requiring a periodic CAM rate of up to ten messages per second [11]-[13]. Hence, solely the establishment of a mutual awareness could saturate and congest the wireless channel, especially according to the following considerations. First, each CAM message could have a size of between 250 and 800 Bytes, because of digital signatures and certificates that secure and authenticate the information contained in those messages. Second, the communication system is expected to cover distances of up to $1000 \mathrm{~m}$. And third, vehicle densities of up to 25 vehicles $/ \mathrm{km} / \mathrm{lane}$ are not an exception (cf. the reported capacity of multilane highways as listed in Table 1. As a result, the total amount of traffic generated per second for mutual awareness could easily exceed the available data rate of $6 \mathrm{Mb} / \mathrm{s}$. As a consequence, the performance of the communication system will degrade significantly if no countermeasures are taken.

\section{A CONTROL THEORY APPROACH}

The process of restricting the load on the wireless channel, and thereby the congestion in the wireless network, and the process of adapting the communications parameters to guarantee a certain awareness level are very closely related to traditional control theory. In particular, due to the shared wireless communication channel and the lack of a centralized coordination entity in vehicular communications, both processes are representatives of the distributed control discipline. For instance, congestion in the network can not be avoided or reduced if only one single node is decreasing its transmission power and/or rate, and more importantly, the result of the selected action can not be observed by the node itself, but only by its neighbors. That implies that all nodes should — at least, for an optimal and reliable control-act cooperatively and provide feedback about the result of their actions to each other. Similarly, the success of an increased transmission power with regard to a desired awareness range can only be determined by the receiving node, and not by the transmitter itself.

Because of the relationship of congestion and awareness control to traditional control theory, this paper discusses existing proposals for congestion and awareness control with respect to the concepts and notions typically used in control theory. For this purpose, both methods are analyzed and compared according to the general framework sketched in Fig. 2: an algorithm might use some sort of detection to classify the traffic situation or scenario

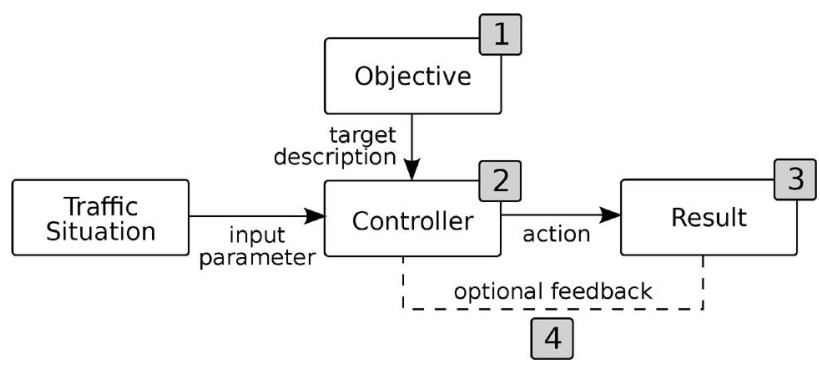

Fig. 2. Analysis of congestion and awareness control algorithms from a control-theory-based perspective. In general a controller adapts the transmission parameters, based on its objective and the detected traffic situation, in order to achieve a particular result. Optionally, the controller makes use of some sort of feedback with regard to the observed result to optimize its performance. Depending on the objective (1), e.g. a network-wide limitation of the channel load or an awareness-range requirement, the scope of the open- or closed-loop controller (2) is either global or local. Consequently, optional feedback (4) about the result can come from the network or from individual nodes only (3). 
which the node is currently in, and which might be used proactively by the controller as feedforward input. The controller itself decides how the transmission will be adjusted, of course depending on the situation and the corresponding target description, i.e. the current objective. The selected action then leads to an observable result, which can be fed back to the controller in order to improve its accuracy.

Based on Fig. 2, particular control algorithms are also classified into open- and closed-loop controllers. The former do not make use of feedback to correct and optimize the decisions made in the past, and typically incorporate a system model to derive the actions to be taken. The advantage of such a control loop is the nonexisting overhead, but, obviously, the performance and robustness of such a controller depends on the accuracy of the system model used. On the contrary, closed loop controllers employ feedback to determine how well the objective has been achieved. An often used closed loop controller is the generic proportional-integral-derivative (PID) controller, which uses the present error $(P)$, the accumulation of past errors $(I)$, a prediction of future errors $(D)$, or only a subset of those measures to control the system. Compared with open loop controllers, closed-loop controllers can improve the control due to the use of feedback data, at the cost of communications overhead. In addition, and particularly if a generic PID controller is used, they do not incorporate any system model, have no direct knowledge of the underlying process and perform poorly in nonlinear systems. With respect to feedback, it is also necessary to distinguish between explicit feedback, i.e., first-order feedback with regard to the desired result, and implicit feedback, i.e., second-order feedback that is obtained by using different observations that are to some extent correlated to the actual observation. A possible implicit feedback could be, for instance, the locally observed number of neighbors or MAC layer reception statistics.

Another important aspect is how the design objective can be quantified and how the achievement of the objective is measured. When awareness control protocols are used, the objective could be defined as the reliability with which a certain vehicle's awareness range is guaranteed. However, for congestion control, the question of how to describe the objective is more difficult to answer, since more than one metric for channel congestion exists, and all have their advantages and disadvantages. For instance, the use of a metric such as the channel busy time ratio, i.e., the fraction of time during which the channel is considered busy by the access layer, or the channel load, i.e., the fraction of time during which the sensed energy exceeds a specific threshold, cannot account for overlapping transmissions, but has the advantage of being easily implementable by the communication hardware. On the other hand, a metric such as the beaconing load [14], though it does quantify the amount of overlapping packets, is not directly measurable by the hardware.

\section{CONGESTION CONTROL}

Congestion control techniques for vehicular communications can be classified according to several criteria. The major classification criterion considers the information base from which congestion control mechanisms derive their decision to adjust the transmission parameters. The first class, which in the literature is sometimes also referred to as reactive congestion control, uses first-order information about the channel congestion status to decide whether and how an action should be undertaken. Because of their nature, actions to lessen channel load are undertaken only after a congested situation has been detected. Using control theory terminology, reactive congestion control approaches can be classified as an instance of feedback control mechanisms. The second class, sometimes also referred to as proactive congestion control, uses models that, based on information such as number of nodes in the vicinity and data generation patterns, try to estimate transmission parameters which will not lead to congested channel conditions, while at the same time providing the desired application-level performance. In particular, such mechanisms typically employ a system model to estimate the channel load under a given set of transmission parameters, and make use of optimization algorithms to determine the maximum transmit power and/or rate setting that will adhere to a maximum congestion limit. Using control theory terminology, proactive congestion control approaches can be classified as an instance of feedforward control mechanisms.

Another criterion used to classify congestion control techniques is what type of information is used to feed the control system, which is typically only locally available information, or also information provided by neighboring vehicles - dubbed distributed information in the following.

Finally, existing solutions can further be classified with reference to the means through which congestion is controlled, which is typically achieved by adjusting the transmission power, the packet generation rate, the carrier sense threshold or a combination of a subset of the transmission parameters.

Let us start briefly discussing the relative advantages and disadvantages of proactive vs. reactive approaches. Given their ability to prevent congestion, proactive approaches are very appealing for vehicular environments, where radio communications are primarily used for safety applications, whose performance would be seriously threatened by congested channel conditions. However, proactive approaches come with two major drawbacks. First, in order to estimate the expected load generated by neighboring vehicles, such approaches require a communication model that maps individual transmission power levels to deterministic carrier sense ranges. However, this mapping is reasonable only as long as it reflects the average propagation conditions of the wireless channel. Thus, propagation conditions should be either dynamically 
estimated as the vehicle moves, which is very difficult to do in a practical scenario, or they should be statistically estimated to build specific profiles for different environments, e.g., urban and highway. A second major drawback of proactive approaches is the need to carefully estimate the amount of generated application-layer traffic in a certain period of time. Although in some cases this is indeed possible (e.g, in the case of applications built on top of periodic beacon exchange), accurate application-layer traffic estimation is a challenging task in general.

Reactive approaches, which do not suffer of the drawbacks that accompany proactive mechanisms, nonetheless have the notable disadvantage of undertaking control actions only after a congested channel condition has been detected. Considering that some time is needed to recover from a congested channel situation, this means that reactive approaches expose safety-related applications to the risk of not being able to fulfill their design goal, due to the poor (temporary) performance of the underlying radio channel. Another disadvantage of reactive approaches is that important design goals such as fairness and packet prioritization are more difficult to achieve than in a proactive approach. We remark that fairness is important in vehicular networks to ensure that all vehicles in the network have similar opportunities to communicating with nearby nodes. In fact, if congestion control were to be obtained by sacrificing, say, a specific node in the network is forced to set its transmission power to a very low value, this node would not have a chance to communicate with nodes in its surrounding, impairing application-level performance. Most importantly, in safety-related applications, every vehicle in the network should be able to receive fresh information about the status of the other vehicles in the surrounding, as well as to communicate its own status to the surrounding vehicles. Hence, fairness becomes a major design goal in safety-related applications. As for prioritization, providing a strict prioritization of different classes of packets is an important requirement for vehicular networking, which is partly addressed in the drafted IEEE 802.11p standard by adopting the enhanced distributed channel access (EDCA) mechanism defined within IEEE 802.11e.

\section{A. Related Work}

Before describing a relevant congestion control approach, we briefly survey the most representative studies aimed at optimizing the packet generation rate and transmission power of beaconing applications. While this body of work is not directly concerned with controlling congestion on the wireless channel, it has the merit of giving very useful insights into the effects of varying rate and transmission power on beaconing performance. These insights can be considered as knowledge base upon which state-of-the-art congestion control approaches are built. Further, with respect to the literal interpretation of congestion control in this paper, those approaches might rather be termed congestion reduction techniques, which, on their own, are of course able to reduce the number of messages transmitted to the channel, but which are not actually able to effectively avoid congested and overloaded channel conditions.

In [15], the authors present a performance evaluation study of cooperative collision warning applications based on periodic beaconing. The major contribution made by this study is the introduction of a novel parameter to measure the performance of cooperative collision warning applications, namely the packet inter-reception time. This metric, defined as the time elapsed between two successful reception events at a vehicle referring to beacons sent by another, specific vehicle, is motivated by the observation that what is relevant for active safety applications is the freshness of the status information gathered from surrounding vehicles. Thus, a few consecutive failed receptions are much more harmful to active safety applications than are several scattered failed receptions. After introducing the novel metric, the authors go on to perform an extensive simulation-based performance study on the effects of using different beacon generation rates and transmission power values on the packet inter-reception time.

In [16], the authors investigate the effect of different beaconing strategies on active safety application performance. More specifically, the authors consider tracking accuracy as the performance metric, which is defined as the error (as perceived by the active safety application) in tracking the positions of neighboring vehicles. After having defined the performance metric, the authors present different beaconing strategies aimed at minimizing tracking error, and identify an adaptive beaconing policy with repetitions as the best performing one. According to this policy, a beacon is sent only if the predicted tracking error of the own position at surrounding vehicles exceeds a threshold. If the threshold is exceeded, the beacon is sent a few times (the number of repetitions is a tunable parameter) to increase the probability that the beacon is correctly received by neighboring vehicles, thus improving tracking accuracy. As mentioned in the beginning of this section, such a mechanism will help to reduce the congestion, but it does not control the congestion of the channel in the first place.

Another example of congestion reduction can be found in [17]. The authors focus on emergency warning messages, that are sent whenever a vehicle shows an abnormal behavior (e.g., it broke down and is blocking the road/lane, or it lost control and it is changing lanes unexpectedly). The authors aim of optimizing the transmission of warning messages is based on the observation that messages should be repeatedly sent out until the "abnormal" behavior stops and the vehicle returns to "normal" behavior. The authors further state that if several abnormal vehicles are sending out emergency warning messages at a constant rate, the average delivery delay will increase rapidly due to channel 
congestion. Consequently, the number of simultaneous emergency warning transmissions should be carefully controlled. To achieve this goal, the authors propose a "multiplicative rate decreasing algorithm," which decreases the retransmission rate of an emergency warning message over time. As a result, several emergency warning messages can be served and delivered by the system with limited delay. The above transmission strategy is further optimized by defining strategies to freeze the generation of emergency messages when certain conditions are met (e.g., redundant transmissions from following vehicles).

According to the terminology defined in Section III, the approach of [17] belongs to the class of proactive approaches, and acts on packet generation rate to prevent congestion. Yet, the approach of [17] is mostly an openloop controller, since the multiplicative rate decreasing algorithm that is used to tune the packet generation rate is based only on predicted performance based on suitable models of the communication channel. On the other hand, a form of primary feedback (e.g., reception of redundant transmissions from following vehicles) is used in the decision rules to freeze emergency message transmission.

Apart from the cited studies above, other congestion reduction solutions that adapt the transmission power and generation rate based on the current velocity exist as well (e.g., [18], [19]). Since the paper focuses on actual congestion control techniques, we will skip their detailed presentation here and instead survey a collection of representative congestion control approaches for cooperative vehicular systems. One of these approaches, called distributed fair-power adjustment for vehicular environments or D-FPAV [20], will be described in detail in the next section.

On the reactive side of congestion control, Khorakhun et al. developed an algorithm that adjusts either the transmission power or the packet generation rate with relation to the locally measured channel busy time ratio [21]. The channel busy time is the fraction of time during which the channel was sensed busy. Depending on whether the local measurement is below or above a predefined threshold, the transmission power or generation rate is either increased or decreased by one step. In order to achieve a higher level of fairness, the authors stated that it is necessary to exchange the local measurements among neighboring vehicles, and allow an increase of the transmission power/rate only if the currently used value is below the average power/rate configuration used by the vehicle's neighbors. Compared with proactive approaches, this reactive approach is not able to avoid congestion on the wireless channel, and supports no prioritization of different classes of messages. In addition, a simple analysis shows that the proposed algorithm is not able to prevent oscillations in the adjustment process. The issue is systematic and fundamental: since not all vehicles perform the transmit power adjustment at the same point in time, it can easily happen that the transmit power reduction at a few nodes leads to a reduced channel busy time observation from the perspective of neighboring nodes that have yet not reduced their transmit power. As a result, those nodes will possibly increase their transmit power (instead of decreasing it as well), and amplify the transmit power reduction of nodes that have already decreased their transmit power. It is obvious that some sort of additional feedback is needed to indicate the reason why the measured channel busy time has decreased or to determine who should reduce first.

A hybrid approach that attempts to combine the advantages of both proactive and reactive approaches was proposed by Baldessari et al. in [22]. Their solution consists of an improved rate control, an improved power control and a combined power and rate control algorithm, all of which use channel busy time observations to derive the number of neighbors in the surrounding area (optionally, also through an additional exchange of local vehicle density estimations). Based on the number of neighbors and a predefined channel busy time threshold, the authors then either derive a packet generation rate directly, or start with a fixed packet generation rate and derive the maximum transmission power which will not violate the threshold. In the latter case, the authors assume that the vehicles in the surrounding area are distributed uniformly and, typical for a proactive approach, make use of a communication model that maps carrier sense ranges to individual transmission power levels.

Another hybrid congestion control approach was recently proposed in [23], where the authors adaptively change both beacon generation rate (in a proactive way) and transmission power (in a reactive way) with the goal of reducing channel congestion, and consequently improving a vehicle's ability to accurately track the position of surrounding vehicles. Two slightly different control approaches are applied to the tuning of beacon generation rate and transmission power. Beacon generation rate is tuned based on a predicted tracking error of own position. The prediction accounts for channel unreliability, i.e., packet losses, by including the observed fraction of successfully received beacons sent by surrounding vehicles. Thus, a closed-loop feedforward controller based on secondary feedback is used for setting the beacon generation rate. Additionally, transmission power control is applied based on the observed channel status (more specifically, based on the channel busy time). This part of the algorithm is thus a closed-loop feedback controller based on secondary feedback. Note that both beacon generation rate and transmission power use information locally available at the vehicles (i.e., direct observations) to control transmission parameters. As a consequence, this mechanism bears the same fundamental issue observed for [21]: without knowing the channel congestion status of the surrounding nodes, the transmission power adaptation 
mechanism cannot know why the channel is no longer congested and which vehicle should reduce or increase its power value first.

\section{B. Contribution}

In this section, we present the D-FPAV approach to proactive, distributed congestion control in vehicular environments. D-FPAV achieves congestion control by varying the node transmission power, where a node's transmit power setting depends on predictions of application-layer traffic and the observed number of vehicles in the surrounding.

D-FPAV is designed to pursue all the optimization goals described in the previous subsection:

1) congestion control: limit the load on the wireless medium in order to prevent congestion generated by application-layer traffic. The metric used to assess the effectiveness of congestion control is channel access time. Furthermore, the authors show the benefits of congestion control on the performance of multi-hop emergency message propagation.

2) fairness: maximize the minimum transmit power value over all transmission power levels assigned to the nodes forming the vehicular network, subject to Goal 1.

3) prioritization: improve the basic IEEE 802.11 EDCA mechanism to provide a better prioritization of higher priority over lower priority messages.

We observe that the D-FPAV approach is aimed at limiting the amount of traffic that is generated by vehicles, with the goal of keeping this load under a specified congestion limit (the MAL value defined in the following). By tuning the congestion limit, the optimal point of the "interference level" versus "reception rate" tradeoff can be found. The optimal tuning of this tradeoff is however out of D-FPAV scope.

The D-FPAV protocol is periodically executed on the nodes forming the vehicular network, in order to adjust node transmission power in response to changes in the network topology or application-layer traffic patterns. Before presenting D-FPAV, we introduce some notation and basic definitions. We denote by $N=\left\{u_{1}, \ldots, u_{n}\right\}$ the set of nodes in the vehicular network. Each of these nodes can set its transmission power in the interval $\left[P_{m}, P_{M}\right]$, where $P_{m}$ is the minimum and $P_{M}$ is the maximum possible transmission power. Given a set $N$ of nodes as above, a power assignment function $P A$ for $N$ is a function that assigns to every node $u_{i} \in N$ a value $P A(i) \in[0,1]$. The power used by node $u_{i}$ to send application-layer messages is $p_{i}=P_{m}+P A(i) \cdot\left(P_{M}-P_{m}\right)$.

For any node $u_{i}$ in the network, we use $C S(P A, i)$ to denote the carrier sensing range of node $u_{i}$ at transmission power $p_{i}=P_{m}+P A(i) \cdot P_{M}$, and $C S(M A X, i)$ to denote the same range at maximum transmission power $P_{M}$.
A fundamental notion in D-FPAV is that of applicationlayer load (AL) generated by a node, and of channel load (CL) experienced by a node under a certain power assignment PA. Formally, $A L(i)$ denotes the application-layer load (expressed in bytes/sec) that node $u_{i}$ is expected to generate in the next period, where the period represents the interval of time before the next D-FPAV execution. Based on these definitions, the CL experienced by a node $u_{i}$ can then be computed based on the AL generated by the nodes in the surrounding as follows:

$$
C L(P A, i)=\sum_{u_{j} \in I(P A, i)} A L(j)
$$

where $I(P A, i)=\left\{u_{j} \in N: u_{i} \in C S(P A, j)\right\}$.

The intuition behind our definition of channel load is that the load observed at $u_{i}$ can be estimated as the sum of the application-layer load generated by nodes in the set $I(P A, i)$, i.e., those nodes having $u_{i}$ within carrier sensing range at the current transmit power levels. This is because a transmission from a node in set $I(P A, i)$ prevents $u_{i}$ from accessing the channel.

The congestion control under fairness constraints (CCF) problem we are attempting to solve is defined as follows:

Definition 1 (CCF Problem): Given a set $N=\left\{u_{1}, \ldots, u_{n}\right\}$ of nodes, and a value MAL of the Maximum Applicationlayer Load admitted on the wireless channel (expressed in bytes/sec), solve the following optimization problem

$$
\left\{\begin{array}{l}
\max _{P A \in \mathbf{P A}}\left(\min _{u_{i} \in N} P A(i)\right) \\
\text { subject to } \\
C L(P A, i) \leq M A L \forall i \in\{1, \ldots, n\}
\end{array}\right.
$$

where $\mathbf{P A}$ is the set of all possible power assignments.

Solving CCF addresses design goals 1 and 2 above, where MAL (a choice of the network designer) is used to control the congestion generated by application-layer load. As we show in the following, goal 3. can be achieved by transmitting low-priority messages using the transmit power computed solving CCF, and by transmitting highpriority messages at maximum power.

The D-FPAV algorithm is reported in Fig. 3, and is composed of the following steps: 1) gather information about AL for nodes within (maximum) carrier sense range; 2) based on 1), locally execute the FPAV algorithm from [14] to compute the optimal CCF solution for the nodes within (maximum) carrier sense range; 3) exchange the locally computed transmission power values with surrounding vehicles; 4) select the minimum transmission power value among the one locally computed and those 
Algorithm D-FPAV: (algorithm for node $u_{i}$ )

INPUT: $\quad \mathrm{AL}$ of all the nodes in $C S(M A X, i)$

OUTPUT: a power setting $P A(i)$ for node $u_{i}$, such that the resulting power assignment is an optimal solution to CCF

$I(M A X, i)$ denotes the set of nodes $u_{j}$ such that $u_{i} \in C S(M A X, j)$

1. Based on the $\mathrm{AL}$ of the nodes in $C S(M A X, i)$, compute the maximum common tx power level $P_{i}$ s.t. the MAL threshold is not violated at any node in $C S(M A X, i)$

$2 a$. Disseminate $P_{i}$ to all nodes in $C S(M A X, i)$

$2 b$. Collect the power level values computed by nodes $u_{j} \in I(M A X, i)$; store the received values in $P_{j}$

3. Compute the final power level:

$P A(i)=\min \left\{P_{i}, \min _{u_{j} \in I(M A X, i)}\left\{P_{j}\right\}\right\}$

Fig. 3. The D-FPAV algorithm.

computed by surrounding vehicles in order to build the network-wide optimal solution to CCF.

The FPAV algorithm of [14] is a centralized algorithm based on the well-known "max-min" principle: the transmission power of all vehicles in a surrounding area is 'virtually' increased step-by-step (starting at lowest possible power level), while estimating the resulting application load at each vehicle after each step. As long as the MAL threshold is not violated at any vehicle, and the maximum allowed transmission power has not been reached, power levels are further increased. Upon termination, FPAV has thus computed the highest common transmission power level which did not violate the MAL parameter in the whole network.

In [20], it is formally proven that D-FPAV computes an optimal solution to CCF under the following assumptions: 1) carrier sense ranges of nodes are symmetric; 2) each node is able to accurately estimate the AL for the next period; and 3) each node is able to gather AL information from all nodes within maximum carrier sense range. In practical scenarios, these assumptions are unlikely to hold, due to the complexity of the propagation environment 1 ), and difficulties in accurately predicting AL and gathering AL information 2) and 3). Yet, in [20] it is shown through extensive simulation that D-FPAV successfully solves the CCF problem at least when assumption 3) is released, i.e., when nodes have only partial knowledge of the AL generated by nodes within maximum $\mathrm{CS}$ range.

In [20], D-FPAV is evaluated in a scenario ${ }^{1}$ in which application-layer load at each node is generated by a beaconing application, which periodically generates packets to report vehicle status to nodes in the surrounding area. Beacon messages are considered low-priority messages in this scenario, and transmitted using D-FPAV computed transmission power with lowest EDCA priority

\footnotetext{
${ }^{1}$ For details on the simulation scenario, including features of the radio environment, please see [20].
}

class. Besides beaconing messages, event-driven emergency messages are randomly generated within the network. These are high-priority messages that are seldom generated, and, given their safety-critical nature, are not subject to congestion control. Emergency messages are sent at maximum transmission power $P_{M}$ using the highest priority EDCA traffic class.

An important issue to understand in the D-FPAV approach is the tradeoff between accuracy of channel load estimation on a vehicle, and additional overhead which is put on the channel. In fact, as the carrier sense range is typically larger than the transmission range, the only way to acquire knowledge about presence of vehicles located outside the transmission range is by making use of a multihop strategy, i.e., having vehicles retransmit the position of their neighbors. Clearly, propagating this information in a multihop manner puts an additional load on the channel, which can be considered as control overhead.

In order to optimally tune the above described tradeoff, the following design decisions have to be made: how often the status of neighboring vehicles should be forwarded, what range of neighbors must be included, and which transmission power must be used to transmit this information.

The following strategies have been considered in [20]: piggyback the aggregated status information (position of surrounding vehicles) to 1) each beacon, 2) every fifth beacon, or 3) every 10th beacon, and transmit it with power $P A(i)$ (the transmit power value as computed by D-FPAV). The authors found that piggybacking aggregate status information in 1 out of 10 beacon messages results in the best compromise between control overhead and effectiveness of congestion control.

The probability of correctly receiving a beacon or emergency message as a function of distance with and without D-FPAV is reported in Fig. 4. As seen from the

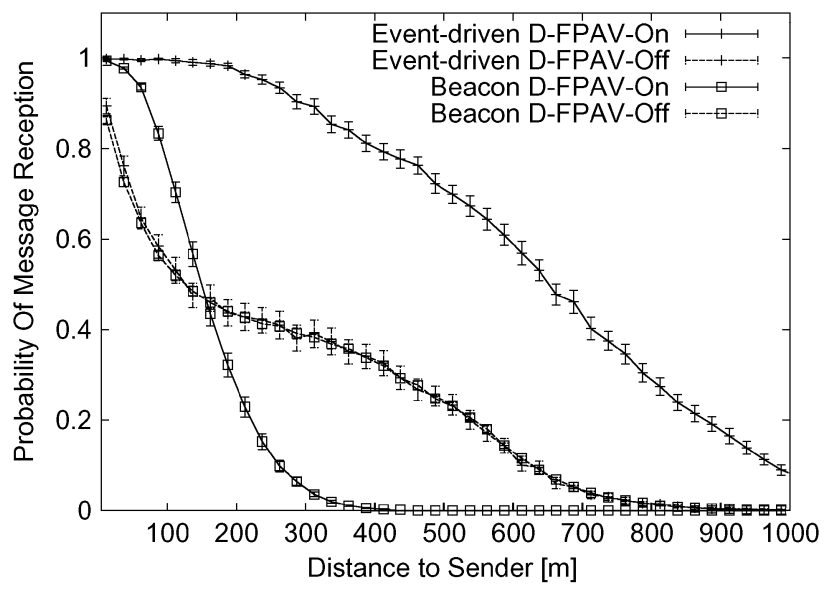

Fig. 4. Probability of successful reception of periodic beacon and emergency messages at varying distances. MAL is set to $2.5 \mathrm{Mb} / \mathrm{s}$. 


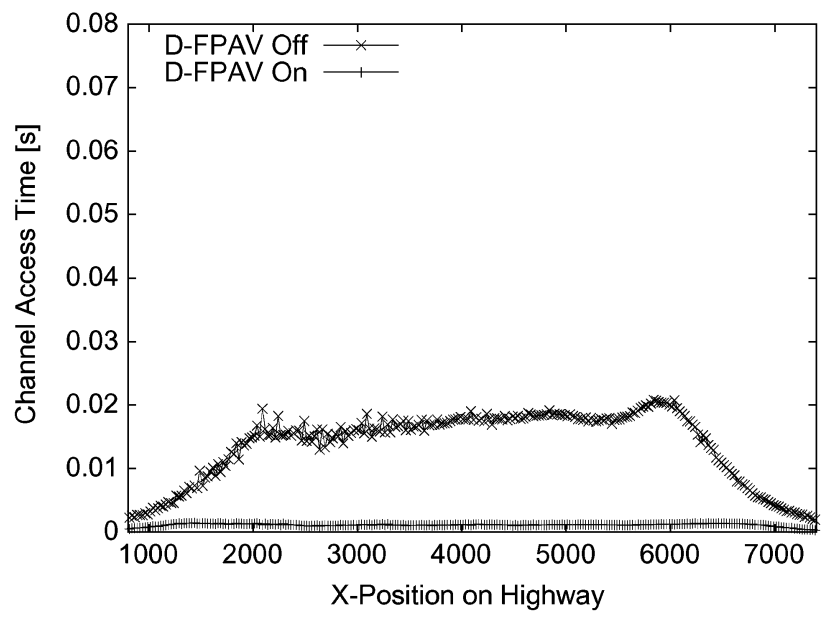

Fig. 5. A verage channel access time experienced by periodic beacon messages as a function of vehicle position on the highway. MAL is set to $2.5 \mathrm{Mb} / \mathrm{s}$.

figure, EDCA alone is not sufficient to clearly prioritize emergency over beacon messages. On the other hand, D-FPAV achieves a clear prioritization of emergency over beacon messages, i.e. emergency messages have a consistently higher reception probability in the whole range of transmission distances. It is also interesting to observe that D-FPAV congestion control mechanism has beneficial effects not only on high-priority traffic, but also on low-priority traffic (beacons): in fact, their reception probability at close distances from the transmitter (within $150 \mathrm{~m}$ ) is considerably higher than when no congestion control exists.

The effectiveness of the D-FPAV approach in achieving fair channel access opportunities is shown in Fig. 5, which reports the channel access time of vehicles as a function of their position on the road: without D-FPAV, channel access time depends highly on the density of vehicles in the surroundings, and it is thus unfairly distributed. On the other hand, when D-FPAV is active, the load generated by the beaconing application is kept under control, and channel access time is nearly constant throughout the network.

In a follow-up study [24], we showed that in order to effectively guarantee a strict enforcement of an upper channel load limit and to provide fairness with respect to channel access opportunities, it is necessary to propagate the position of neighbor vehicles for at least two hops. As described above, the D-FPAV protocol provides this information by piggybacking this information only in 1 out of 10 beacon messages in order to reduce the overhead, yet, the overhead can still grow to $40 \%$ - compared to the actual AL data. In [24], we therefore developed a distributed algorithm that adjusts the transmission power based on averaged values for the neighbor information instead of using detailed neighbor information about each single node. By using only this averaged information, we were able to reduce the overhead down to less than $1 \%$, at the cost of only slighty exceeding the pre-defined MAL limit.

\section{Standardization}

In Europe, congestion control in vehicular communications is considered to be a building block and seen as mandatory in order to guarantee a reliable communication performance for safety-related applications. This was acknowledged by the Car-2-Car Communication Consortium (C2C-CC) as early as in 2009 through the establishment of a task force on transmit power control. The results from this task force have helped to persuade the European Commission to establish a specialist task force (STF) on the configuration and validation of decentralued congestion control methods for intelligent transportation systems (ITS) and techniques to enable the co-existence of cooperative ITS and dedicated short range communication (DSRC) within the European Telecommunications Standard Institute (ETSI). Since March 2010, the STF with its six experts from the industry and research community have been working on a technical specification for a standardized congestion control algorithm to be used by ITS.

The specification defines a mandatory basic congestion control algorithm based on a controller that uses no feedback and only information that is locally available. In addition, the specification describes an enhanced control algorithm that uses feedback from neighboring nodes, e.g., their observed channel load and their currently used transmit power. Both approaches will use the channel load metric to define the congestion limit, since it can be implemented by the hardware.

\section{AWARENESS CONTROL}

Following the previous discussions, awareness control techniques are aimed at ensuring each vehicle's capacity to detect as well as to communicate with the relevant vehicles in their local neighborhood. Awareness control protocols are needed to reliably and efficiently support higher layer protocols and applications, for example, ensuring that traffic safety applications obtain, at a minimum, the level of awareness that is required to detect dangerous traffic situations in advance and act accordingly.

Cooperative vehicular systems impose very stringent application requirements, with most of the applications being supported by the periodic exchange of beacons. It is generally assumed in the literature that the cooperative application requirements can be defined in terms of dissemination area (or range), latency (or delay) and reliability [25]. This extends the preliminary requirements defined by VSC [26] and ETSI [27], which consider only some of these requirements. While the dissemination area can be defined as the geographic area where a given message should be received, the latency is the maximum 
allowed delay to deliver such message, and the reliability is the minimum probability of receiving such a message (usually estimated during a certain time window). The requirements imposed by cooperative applications represent the basis of awareness control protocols.

\section{A. Related Work}

Initial studies have been conducted to evaluate the communications and applications performance in VANETs, and serve as fundamental studies for the design of awareness control protocols. For example, the work in [28] presents a performance and sensitivity analysis of different MAC layer protocols, based on the idea of repetitive transmissions over CSMA. In this work, the authors propose that a packet be retransmitted multiple times during its lifetime, and study different repetition mechanisms by means of simulation and a detailed mathematical anlalysis. The conducted study identifies the operating and communications conditions (number of interferers and packet generation rates) under which the application requirements are satisfied and the channel load is maintained under a certain limit. In [15], the authors conduct a performance evaluation study of cooperative collision warning applications under different traffic densities, and explore different packet generation rates and transmission ranges. The conducted study shows the importance of considering appropriate metrics to evaluate the performance of cooperative applications, such as latency or packet inter-reception time. This observation results from the importance of the freshness of the information received from surrounding vehicles. The relevance of adequately evaluating the performance of cooperative vehicular systems has also been emphasized in other studies. For example, the work in [29] highlights the need to differentiate communication and application performance or reliability. In particular, in [29] the authors demonstrate the suitability of cooperative vehicular systems to improve traffic safety based on real-world experimental data on highways.

Following the control-theory perspective discussed in Section III, awareness control protocols can be classified as open-loop or closed-loop approaches, and can use implicit or explicit feedback. Different existing open-loop awareness control protocols use power-range maps to dynamically adapt each vehicle's transmission power as a function of its transmission range requirements. For example, the work in [30] proposes an OPportunistic-driven adaptive RAdio resource Management (OPRAM) mechanism, that adapts each vehicle's transmission parameters to reliably and efficiently exchange a message before reaching a critical safety area, for example an intersection. The OPRAM mechanism is an application-driven awareness control protocol that is based on radio propagation estimates to dynamically calculate the required transmission power levels as a function of the distance to the critical safety area. Despite having been designed as a power-range map based technique,
OPRAM could be evolved to dynamically adapt the transmission power and packet generation rate to the experienced channel load, in order to compensate for the negative effects of packet collisions on the application's reliability. OPRAM will be described in detail in the next section.

Multihop beaconing protocols represent an alternative open-loop solution for awareness control. With these protocols, broadcast messages transmitted by a vehicle are relayed by neighboring vehicles to achieve the target probability of reception at high distances within the required delay. In [31], the multihop vehicular broadcast (MHVB) protocol is intended to efficiently relay broadcast packets over multiple hops, and satisfy the target dissemination area within the allowable latency. With MHVB, only the vehicle that correctly receives a given broadcast message and is located at the highest distance from the transmitter will relay such message. A similar approach was proposed in [32], where a vehicle can relay multiple beacons during its lifetime in a single packet as long as it has received each one of these beacons less than a established maximum number of times. With multihop beaconing protocols, the beacon's transmission power was able to be reduced compared to single hop beaconing protocols. In this context, the work in [33] compares single-hop and multihop beaconing protocols. This study shows that under simplified propagation and multihop operating conditions, the channel load in multihop beaconing protocols can be reduced using packet multiplexing techniques. With these techniques, when a vehicle has to relay a broadcast packet it will attach its own broadcast packet to the relayed message. However, in realistic environments where packets can be lost due to radio channel errors and packet collisions, the reduction of the channel load obtained with multihop beaconing cannot be achieved. It is worth mentioning that, in scenarios where obstacles block the radio signal, such as buildings or trucks, multi-hop beaconing protocols could be required to successfully satisfy the application requirements.

Closed-loop awareness control protocols make use of exchanged broadcast messages to dynamically adapt to the varying propagation and channel load conditions. In some existing solutions, the feedback is implicitly obtained from received messages without the need to transmit extra information. An example of closed-loop solution with implicit feedback is the work in [34], in which the authors propose the use of the received messages to estimate in real-time the path loss or average signal attenuation, by subtracting the power estimated at the receiver from the transmitted power (included by default in the header of all beacons in cooperative vehicular systems). The proposed algorithm dynamically selects the power and data rate required to successfully transmit a packet to a given vehicle, while minimizing the interference generated to other vehicles. In the joint rate-power control algorithm proposed in [23], two different control approaches are applied to adapt the packet generation rate and the transmission 
power. In order to detect abnormal driving maneuvers in advance, the packet generation rate is dynamically adapted to bound the longitudinal and lateral position tracking errors of surrounding vehicles. A new packet is transmitted when the estimated tracking error of surrounding vehicles exceeds a certain threshold. To estimate the tracking error, the algorithm takes into account the channel reliability by dynamically estimating the packet error probability from the packets received from surrounding vehicles. The transmission power is adapted based on the observed channel status. Considering $L_{\min }$ and $L_{\max }$ as the lower/upper transmission range bounds dictated by the safety applications, the transmission range is linearly adapted between $L_{\min }$ and $L_{\max }$ as a function of the experienced channel load. The transmission power is then calculated considering power-range maps based on empirical measurements.

Other closed-loop awareness control solutions are based on explicit feedback. In this case, vehicles inform each other about the correct reception of messages at a certain distance. Using this explicit information, vehicles can decide whether they should increase or decrease their transmission power and/or packet generation rate. For example, the work in [35] includes the target range of the packet, and the IDs of the nodes from which a message was successfully received when their separation distance was larger than the target range, as extra information in the beacon's header. When a vehicle receives more than $N$ beacons containing its ID, it decreases its transmission power, since at least $N$ vehicles beyond the target range are receiving its broadcast messages. This type of closed-loop technique depends heavily on the correct reception of a beacon to adequately adapt its operational parameters. As a result, if some of these messages are not correctly received, for example due to packet collisions under high traffic densities, the vehicles can incorrectly increase their transmission power and augment packet collisions.

The accuracy or precision of the awareness information received from surrounding nodes is also analysed in different protocols proposed in the literature. A representative example is reported in [16], where the authors propose an open-loop approach through which the packet generation rate is dynamically adapted to bound the tracking errors of surrounding vehicles. To this end, a new packet is transmitted by a vehicle only when its movement changes (speed, heading, etc.). In this case, the authors propose that each of these packets be retransmitted several times to ensure its reception by surrounding vehicles, which results in a decrease of the tracking error in realistic propagation environments, at the expense of increasing the channel load. In fact, as shown in [36], the increase of the packet generation rate can augment the probability of successfully receiving a packet at the target distance within the required time window, as long as the channel load is maintained at reasonable levels. In particular, the work reported in [36] proposes the rapid rebroadcasting of each packet during its lifetime to increase its probability of
Basic application-driven awareness control approach INPUT: Established application reliability $p_{a p p}$

OUTPUT: A power and rate setting for vehicle $i$, such that the resulting assignment satisfies the application requirements

1. Update positioning and movement information.

2. Adapt application requirements.

3. Obtain required power and rate based on $p_{a p p}$ and application requirements.

Fig. 6. Basic application-driven awareness control approach.

reception, extending the work presented in [28]. To this aim, various rebroadcasting schemes are proposed based on different open-loop strategies: synchronous and asynchronous designs, repetition with and without carrier sensing, fixed number, and $p$-persistent repetition. A different perspective is reported in [37] with regard to the accuracy of the awareness information required by each vehicle. The work in [37] considers that the packet generation rate should depend on each vehicle's mobility characteristics, and those of the vehicles surrounding it, as well as the traffic context/situation. In particular, the authors propose the use of situation-adaptive beaconing to achieve adequate levels of accuracy or updated awareness information received from neighboring vehicles.

\section{B. Contribution}

The consideration of application requirements in the design of awareness control protocols for cooperative vehicular systems is particularly important due to the critical nature of safety applications. A basic application-driven awareness control approach is summarized in Fig. 6. Its operation is based on each vehicle's application requirements. As an example, in the case of an intersection collision warning application, the requirements would correspond to the minimum distance to the intersection at which two potentially colliding vehicles need to exchange a message to alert the driver with sufficient time to avoid the accident. Since such requirements depend heavily on the vehicles' position, speed and acceleration, each vehicle would need to continuously adapt its application requirements based on its positioning and movement information. Once the application requirements have been updated, each vehicle will accordingly modify its communications parameters (e.g. transmission power and packet generation rate) to satisfy them with certain reliability imposed by the application $\left(p_{\text {app }}\right)$. The adaptation of the communications parameters could be based on some of the protocols and algorithms described in the previous section. While this basic approach could satisfy the required vehicle's awareness level, the following sections describe two application-driven awareness control 


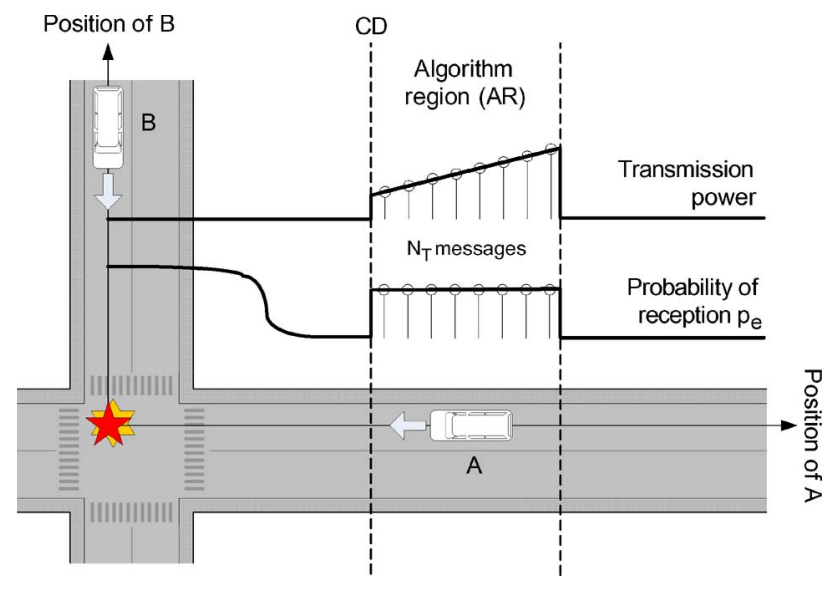

Fig. 7. OPRAM operation in intersection scenarios.

approaches aimed at further improving the communications efficiency through the use of geographic and traffic context information.

1) Geo-Opportunistic Approach: OPRAM [30] is an example of an application-driven awareness control technique aimed at efficiently adapting each vehicle's communications parameters (transmission power and packet generation rate) to guarantee the transmission range and reliability requirements imposed by traffic applications. To further improve the efficiency of the basic awareness control approach previously discussed, OPRAM proposes a geo-opportunistic approach that makes use of the geographic positioning and the knowledge of potentially critical safety areas. To illustrate its operation and benefits, an intersection collision warning application is considered here. However, OPRAM's operation could be easilly adapted to other applications such as cooperative merging assistance and left turn assistance applications.

Intersection collision warning applications in urban intersections represent one of the most challenging scenarios for awareness control protocols due to the strict traffic safety application requirements and the challenging nonline-of-sight propagation conditions. In a typical intersection scenario, two approaching vehicles A and B might collide at the intersection due to the driver's lack of attention, wrong/hidden traffic signals, or any other reason that could provoke the accident despite the driver's ability and perception capabilities (see Fig. 7). To detect each other's presence, the two vehicles periodically broadcast beacon messages. The intersection collision warning application requires that both vehicles exchange at least one packet before the critical distance $(C D)$. The $C D$ distance is the minimum distance to the intersection at which a vehicle needs to receive a broadcast message from a the potentially colliding vehicle to alert the driver of a potential road danger with sufficient time to react, and stop before reaching the intersection. The $C D$ distance typically depends on the vehicle's speed, the driver's reaction time and the vehicle's emergency deceleration. The presence of buildings may require the use of high transmission power levels and/or packet generation rates to guarantee the communication between the two vehicles before the target distance $C D$, and hence avoid the potential accident. However, the constant use of high transmission powers and rates by multiple vehicles could create channel congestion, and increase the system's instability. To reduce the channel load while satisfying the application requirements, OPRAM is designed to dynamically increase the transmission power and packet rate of each vehicle only in a small region before $C D$, called the Algorithm Region $(A R)$, as illustrated in Fig. 7. With this geo-opportunistic increase, OPRAM aims to guarantee with high probability the correct reception of at least one packet from a potentially colliding vehicle before reaching $C D$, while minimizing the overall channel load. Outside $A R$, OPRAM operates with a low transmission power, sufficient to communicate with the vehicles moving along the same street in line-of-sight propagation conditions. The definition of the AR region allows OPRAM to adapt its communication parameters only when approaching a critical safety area, such as an intersection, lane merging zones, entrance ramps, blind curves, etc.

To define the operation of the OPRAM mechanism, we consider that each vehicle transmits $N_{T}$ broadcast packets in AR. Avoiding the intersection collision requires that at least one of these packets is correctly exchanged with high probability before $C D$. Considering the challenging and probabilistic radio propagation conditions, OPRAM has been configured to successfully receive at least one of these messages from a potentially colliding vehicle before reaching $C D$ in $99 \%$ of the cases $\left(p_{\text {appA }}=p_{\text {appB }}=0.99\right)$. An intersection collision could be avoided if at least one of the two vehicles receives a broadcast message with sufficient time to react. If we assume that the success of transmission from vehicle A to vehicle B is independent of the success of transmission from $\mathrm{B}$ to $\mathrm{A}$, the overall application's reliability could be then $p_{\text {app }}=1-\left(1-p_{\text {appA }}\right)\left(1-p_{\text {appB }}\right)=$ 0.9999. However, it is important to note that such full independence is difficult to achieve despite the potentially different interference conditions experienced by each vehicle, and the different obstacles present in their respective local environments. To reach the target reliability, OPRAM initially considers that the probability that a single packet will be successfully received by the potentially colliding vehicle $p_{e}$ is constant and independent in $A R$. Having defined $N_{T}$ and each vehicle's application reliability, such $p_{e}$ probability can be calculated through a Binomial distribution constructed by $N_{T}$ Bernoully experiments [30].

To dynamically calculate the required transmission power level for each of the $N_{T}$ packets transmitted in $A R$, the initial OPRAM implementation considers an open-loop approach, based on propagation models obtained through 
empirical radio channel measurements conducted under the European WINNER project [38]. In particular, OPRAM computes the transmission power for each of the $N_{T}$ packets based on the current distance to the intersection, and the path loss, shadowing and multipath fading propagation effects. ${ }^{2}$ The transmission power is then selected so that each of the $N_{T}$ packets transmitted within $A R$ is received with a probability $p_{e}$. This will ensure that at least one of the $N_{T}$ transmitted packets will be correctly received with probability $p_{\mathrm{appA}}$ (or $p_{\mathrm{appB}}$ ). It is interesting to note that the increase of $N_{T}$ reduces the required $p_{e}$ and the consequent required transmission power levels for a given application reliability.

Under realistic operating conditions, the probability of packet reception from a potentially colliding vehicle would depend not only on the radio channel propagation effects, but also on the channel load, and consequent packet collisions. Packet collisions reduce the probability of packet reception $p_{e}$, and hence decrease the application's reliability. The work in [39] proposed two different packet collisions compensation techniques. These techniques are based on the evaluation of the experienced channel load, and the consequent adaptation of each vehicle's transmission power or packet generation rate in $A R$ to combat the negative effect of packet collisions on the OPRAM performance. With these compensaiton policies, OPRAM could therefore be extended to follow a closed-loop approach based on the feedback received from neighbouring vehicles.

The OPRAM technique was initially designed considering that the $N_{T}$ transmitted messages are received independently. However, such independence cannot be guaranteed under correlated radio channel conditions. Although such correlation effects can be simplified for certain system level investigations, their impact on the instantaneous performance of cooperative vehicular systems cannot be neglected, in particular for critical traffic safety applications. In this context, the work in [40] proposes and evaluates various compensation policies that can efficiently overcome the negative communication effects caused by the radio channel correlation.

For the scenario illustrated in Fig. 7, Fig. 8 depicts the cumulative distribution function $(\mathrm{CDF})$ of the distance to the intersection at which the first message from a potentially colliding vehicle is received, considering the use of OPRAM and the constant transmission power level $(P t=2 \mathrm{~W})$ needed to ensure the same application's reliability under the same operating conditions. As it can be observed, using OPRAM ${ }^{3}$ or a $2 \mathrm{~W}$ fixed transmission power can guarantee that at least one message is exchanged before $C D$ with the target reliability. However, as demonstrated in [41], the application of OPRAM at a system scale results in a more efficient

\footnotetext{
${ }^{2}$ For details on the calculation methodology, please see [30].

${ }^{3}$ The same OPRAM performance is achieved for different $N_{T}$ values.
}

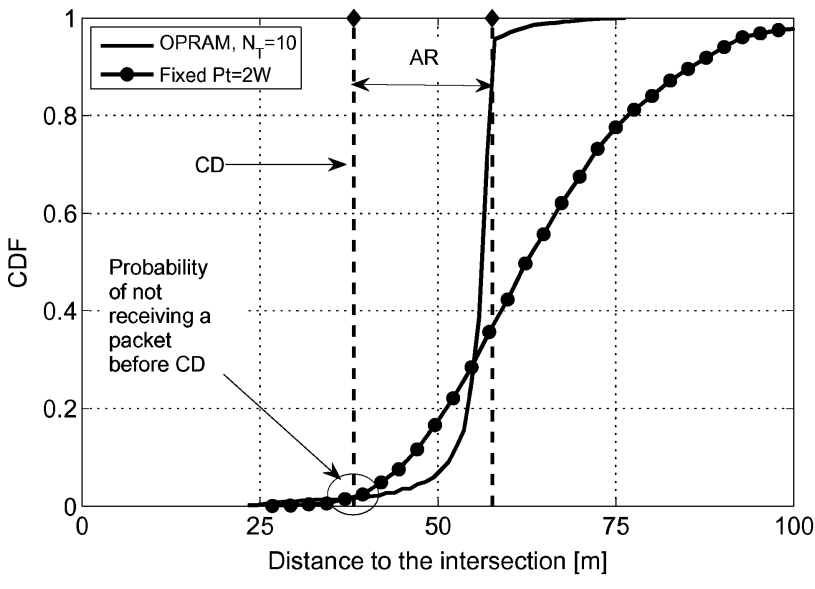

Fig. 8. CDF of the distance to the intersection at which the first message from a potentially colliding vehicle is received.

use of the radio channel, since it is able to considerably reduce the channel load (by nearly 70\%), while guaranteeing the awareness level needed to ensure the same application reliability as with fixed transmission power policies.

2) Traffic Contextual Approach: The previous subsection illustrated the benefits of enhancing basic applicationdriven awareness control approaches through the use of geographical information. This subsection is aimed at demonstrating that the operation of basic awareness control techniques can also be improved through the use of traffic context information. To this end, a lane change assistance application in highway scenarios was considered. This application informs the driver about whether a potential lane change maneuver can be performed in a safe way or not based on the proximity of other vehicles. Such proximity can be detected through the reception of broadcast messages transmitted by neighboring vehicles. Following

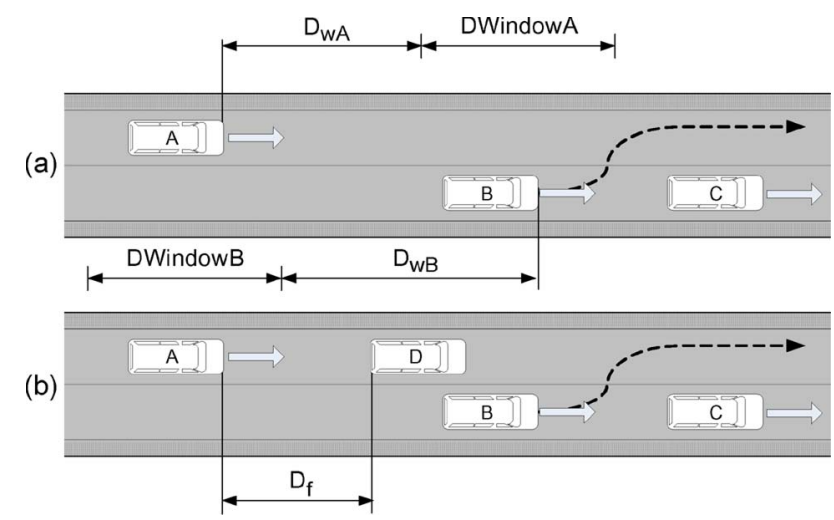

Fig. 9. Lane change assistance application. (a) Scenario. (b) Traffic contextual information. 
the illustration in Fig. 9(a), vehicle B would consider its lane change unsafe if another vehicle A was approaching on the left lane and they were closer than a certain distance $D_{w}$ (Warning Distance). $D_{w}$ represents the minimum separation distance between the two vehicles allowing vehicle B to change lane without making vehicle A reduce its speed, and can be computed as

$$
D_{w}=-\frac{1}{2} \frac{\left(v_{B}-v_{A}\right)^{2}}{a_{A}-a_{B}}+L+D_{s}
$$

where $v_{A}$ and $v_{B}$ represent the vehicles speed in $\mathrm{m} / \mathrm{s}, a_{A}$ and $a_{B}$ their acceleration in $\mathrm{m} / \mathrm{s}^{2}, L$ is the vehicle length in $\mathrm{m}$, and $D_{s}$ is the safety distance. It is important to note that neither of the two vehicles knows the speed of the other vehicle before receiving its first message. Consequently, they need to assume the worst case scenario in terms of speed to calculate their respective $D_{w}$. This corresponds to vehicle A calculating $D_{w}$ considering that vehicle $\mathrm{B}$ is moving at the minimum speed allowed on the road, and it has the lowest possible acceleration in the overtaking maneuver $\left(v_{B}=v_{\min }, a_{B}=a_{\min }=1 \mathrm{~m} / \mathrm{s}^{2}\right)$. Vehicle B will consider that vehicle $\mathrm{A}$ is moving at the maximum constant speed allowed on the road $\left(v_{A}=v_{\max }, a_{A}=0 \mathrm{~m} / \mathrm{s}^{2}\right)$. This results in different $D_{w}$ distances for vehicles with different driving context situations. It is interesting to note that vehicles with a speed outside the $\left(v_{\max }, v_{\min }\right)$ limits could be configured to transmit with a higher transmission power to warn surrounding vehicles with enough time for the driver to avoid dangerous situations. Since only a few vehicles would be driving with a speed outside the limits, this results in a more efficient use of the radio channel than considering all vehicles calculating $D_{w}$ based on speeds higher than $v_{\max }$ or lower than $v_{\min }$.

Based on the proposed application and previous definitions, $D_{w}$ is the minimum distance at which vehicles A and B would need to communicate to avoid a dangerous situation. As a result, $D_{w}$ represents the application requirement according on the basic application-driven awareness control approach previously described. Following this basic approach, each vehicle autonomously adapts $D_{w}$ based on its own vehicular speed. The transmission power is accordingly adapted to satisfy the target reliability following the OPRAM transmission power estimation methodology. In this case, the application reliability has been defined as the probability of receiving at least one broadcast message before $D_{w}$ and during a given time window TWindow (see Fig. 9(a), where the TWindow is mapped to the DWindow distance following the vehicle's speed). To combat the negative effects of packet collisions and radio channel correlation, the compensation policies proposed in [39] and [40] could be considered. However, reduced correlation levels have been observed in highway scenarios [42], and the channel

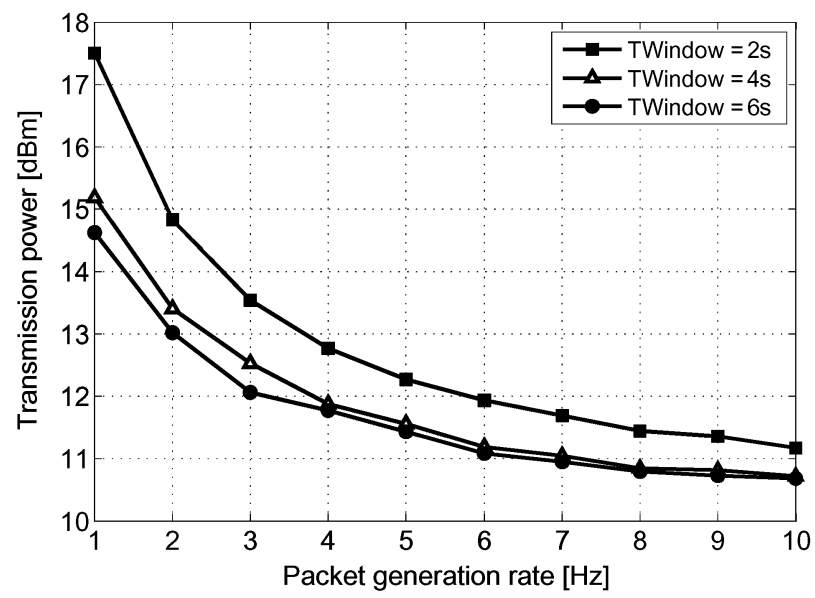

Fig. 10. Communications configurations that satisfy the application requirements with the target reliability $\left(D_{w}\right.$ and $\left.p_{\mathrm{app} A}=p_{\mathrm{appB}}=\mathbf{0 . 9 9}\right)$, considering a traffic density of 15 vehicles/ $/ \mathrm{km} / \mathrm{lane}$.

correlation compensation techniques have not been required in this case. Following this basic approach, each vehicle is able to autonomously and dynamically configure its transmission power to the minimum value that satisfies the application reliability.

Considering a highway scenario with six lanes, the combination of transmission power and packet generation rate that allows meeting the application requirements with the target reliability is illustrated in Fig. 10. In particular, the transmission power levels shown in this figure correspond to the vehicles experiencing the highest $D_{w}$, i.e., vehicles moving at $v_{\max }=120 \mathrm{~km} / \mathrm{h}$ and $v_{\min }=60 \mathrm{~km} / \mathrm{h}$. In this case, the application's reliability for each vehicle has been set to $p_{\text {appA }}=p_{\text {appB }}=0.99$. In this scenario, the multipath fading effect has been modeled by a Nakagami model, following the observations for highway scenarios in [42]. As shown in this figure, when increasing the packet generation rate, the transmission power can be decreased to maintain the same application reliability.

To reduce the channel load and unnecessary interference, the described basic awareness control approach can be improved through the use of traffic context information following a closed-loop approach. To this end, each vehicle could utilize the specific positions of neighbouring vehicles to reconfigure its application requirements and the resulting transmission parameters. An example of the use of traffic context information for the lane change assistance application is illustrated in Fig. 9(b). Considering the previously explained approach, vehicle A would broadcast its beacon message at its $D_{w A}$ distance. However, if vehicle $A$ is aware of the presence of vehicle $D$ through the reception of one of its beacon messages, it can assume that vehicle $B$ would have been informed by vehicle $D$ that it cannot conduct a lane-change maneuver. As a result, if 


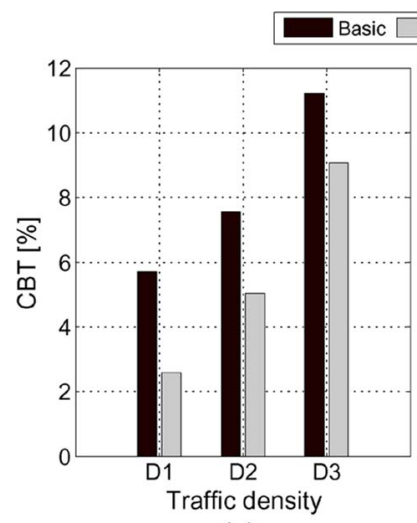

(a)

Fig. 11. Channel busy time for the communications configuration that satisfy the application requirements with the target reliability ( $D_{w}$ and $p_{\text {appA }}=p_{\text {appB }}=0.99$ ), considering a payload of 500 Bytes. (a) $2 \mathrm{~Hz}$ and (b) $10 \mathrm{~Hz}$.

vehicle $\mathrm{D}$ is located at a distance lower than $D_{w A}$ from vehicle $A$, then vehicle $A$ does not need to transmit with the power level required to guarantee $p_{\text {app }}$ at $D_{w A}$. In this context, vehicle A can reduce its transmission power to that needed to communicate with vehicle $\mathrm{D}$, located at a distance $D_{f}$ from vehicle A [see Fig. 9(b)]. Consequently, each vehicle can configure its transmission parameters based on the minimum of the $D_{w}$ and $D_{f}$ distances. This results in vehicle A configuring its transmission power to directly communicate with vehicle B only when there is no vehicle $\mathrm{D}$ in the same lane ahead located at $D_{f}<D_{w}$. Therefore, the use of traffic context information obtained through the periodic exchange of broadcast messages allows the reduction of unnecessary interference and limits the channel load with respect to a basic approach.

Fig. 11 shows the average channel busy time for the basic and traffic contextual approaches previously described. The results shown in the figure have been obtained for a highway scenario with six lanes, different traffic densities $(D 1=7.2$, $D 2=9.6$, and D3 = $14.4 \mathrm{veh} / \mathrm{km} /$ lane) and packet generation rates of $2 \mathrm{~Hz}$ and $10 \mathrm{~Hz}$. It is important to note that all the configurations reported in this figure were able to satisfy the target application's reliability. As it can be observed, the traffic contextual extension of awareness control policies can significantly reduce the channel load (more than 50\% in some cases) while guaranteeing the application requirements. The obtained results also demonstrate that the reduction of the packet generation rate can also decrease the channel load generated, despite the fact that it would require a higher transmission power to meet the application requirements (see Fig. 10).

\section{OUTLOOK}

The design of future cooperative vehicular systems could certainly require the joint consideration of congestion and awareness control protocols. Under certain conditions, the congestion control limitations could prevent the proper function of multiple applications running on neighboring vehicles. This could arise in scenarios such as the one illustrated in Fig. 1. In this scenario, the requirements of the lane change assistance application run by the vehicles under free flow conditions would be notably different from the requirements of the applications run by the vehicles in the traffic jam. While awareness control protocols would adapt each vehicle's communications parameters to efficiently satisfy their individual requirements, congestion control protocols would limit the channel load generated, given the high density of vehicles in the scenario. As a result, the requirements of all the different applications might not be simultaneously satisfied. This example indicates the obvious challenge of how to integrate both control aspects into one system, in particular if the selected actions and adjustments are contradictory. The fundamentally different objectives lead to the issue that a joint realization might be difficult to realize or even mutually exclusive. One potential approach to address this problem is based on the use of additional policies to prioritize among different applications and control the amount of information sent to the wireless channel. An example of this type of policy was proposed in [43], based on application-specific utility functions and a prioritization and rescheduling technique.

In addition to the joint consideration of congestion and awareness control, future cooperative vehicles might need to run different applications simultaneously. As a result, they should be able to simultaneously support potentially different (and maybe contrary) communication and application requirements. How to efficiently satisfy these requirements while efficiently using the communications channel is a challenging aspect that would need to be carefully investigated in the coming years. Considering only safety applications for illustration purposes, these applications might need to detect or monitor neighboring vehicles in the various safety areas shown in Fig. 12. For the example shown in this figure, vehicle A might need to simultaneously run a cooperative forward collision warning (CFCW) application with vehicle $\mathrm{B}$, and an overtaking vehicle warning (OVW) application with vehicle $C$ [27]. In this context, vehicle A would experience a very different relative speed with vehicles $\mathrm{B}$ and $\mathrm{C}$ due to their opposite directions. This would result in very different warning distances and

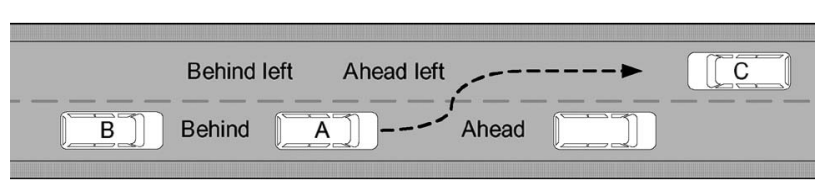

Fig. 12. Multi-application scenarios. 


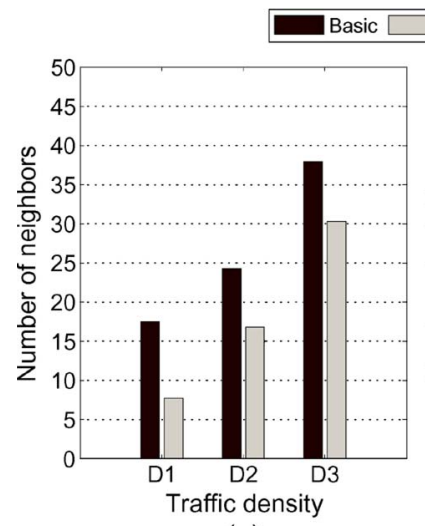

(a)
Traffic contextual

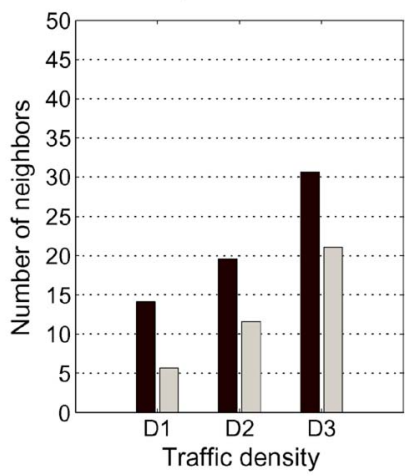

(b)
Fig. 13. Minimum number of neighboring vehicles detected during $\mathbf{9 9 \%}$ of the time for the communications configuration that satisfies the established application reliability $p_{\text {appA }}=p_{\text {арpв }}=0.99$, considering a payload of 500 Bytes. (a) $2 \mathrm{~Hz}$ and (b) $10 \mathrm{~Hz}$.

communications settings for the two simultaneous applications run by vehicle A.

In this context, it is also worth highlighting the necessity of jointly taking into account the requirements of safety and nonsafety applications. For example, considering only the requirements of traffic safety applications in the design of awareness control protocols could compromise the connectivity requirements of nonsafety applications employing multihop transmissions. This example can be illustrated with the highway scenario considered in Section V-B2. In this scenario, the vehicles adapt their communication parameters to support the lane change assistance application. As demonstrated in Section V-B2, the use of a traffic contextual approach satisfying the safety requirements can reduce the risk of channel congestion. However, the decrease of the transmission power without considering the requirements of other applications could compromise, for example, the connectivity requirements. This effect can be observed in Fig. 13. This figure shows the minimum number of neighboring vehicles that each vehicle has in its neighbor list $99 \%$ of the time. The results shown in this figure correspond to the basic applicationdriven awareness control technique previously explained and its traffic contextual adaptation. Following [44], a vehicle is removed from another vehicle's neighbor list after $5 \mathrm{~s}$ without receiving any 1-hop broadcast packet from it. As it can be observed, the use of a contextual approach to reduce the risk of channel congestion reduces the number of neighboring vehicles, which could compromise the connectivity requirements of the different vehicles.

To efficiently support various simultaneous applications, each vehicle should dynamically define the minimum communications parameters (e.g., transmission power and packet generation rate) that are able to satisfy the requirements of each application, following the awareness control proposals discussed in this paper. Then, the communication requirements should be efficiently and safely combined to minimize the channel load generated and satisfy the requirements of all the different applications. To this end, the definition of a communications adaptation layer (CAL) would be needed, and its operation could be as follows. Assume that all applications require the same information to be sent/ received in the periodic broadcast packets transmitted in the communications channel. Let us further assume that a given vehicle is running $N$ applications, each of them with communication requirements $P t_{i}$ (transmission power) and $R_{i}$ (packet generation rate), with $1 \leq i \leq N$. To satisfy the $R_{i}$ requirements of the different applications, the total number of packets transmitted per second by this vehicle would be:

$$
R=\max \left(R_{1}, R_{2}, \ldots, R_{N}\right)
$$

To satisfy the $P t_{i}$ requirements of the different applications, these applications should first be ordered as a function of their transmission power requirements so that $P t_{1} \geq P t_{2} \geq \ldots \geq P t_{N}$. Then, the transmission power of the $R$ packets transmitted per second could be distributed as indicated in Table 2 , so that at least $R_{i}$ packets per second are transmitted with a transmission power equal or higher than $P t_{i}$. An example of the operation of the CAL is provided in Fig. 14, where three applications are being run by a vehicle, and each of them has different transmission power and packet generation rate requirements. Following the proposed adaptation, $R=5$ packets would need to be transmitted per second to satisfy the

Table 2 Communications Adaptation Layer Configuration for Multi-Application Scenarios

\begin{tabular}{|cl|}
\hline $\begin{array}{c}P t_{i}, \text { transmission } \\
\text { power }[\mathrm{dBm}]\end{array}$ & $\begin{array}{l}n_{i}, \text { number of packets transmitted } \\
\text { per second using } P t_{i}\end{array}$ \\
\hline$P t_{1}$ & $n_{1}=R_{1}$ \\
\hline$P t_{2}$ & $n_{2}=\max \left(0, R_{2}-n_{1}\right)$ \\
\hline$P t_{3}$ & $n_{3}=\max \left(0, R_{3}-n_{2}-n_{1}\right)$ \\
\hline$\ldots$ & $\ldots$ \\
\hline$P t_{N}$ & $n_{N}=\max \left(0, R_{N}-n_{N-1}-n_{N-2}-\ldots-n_{1}\right)$ \\
\hline
\end{tabular}




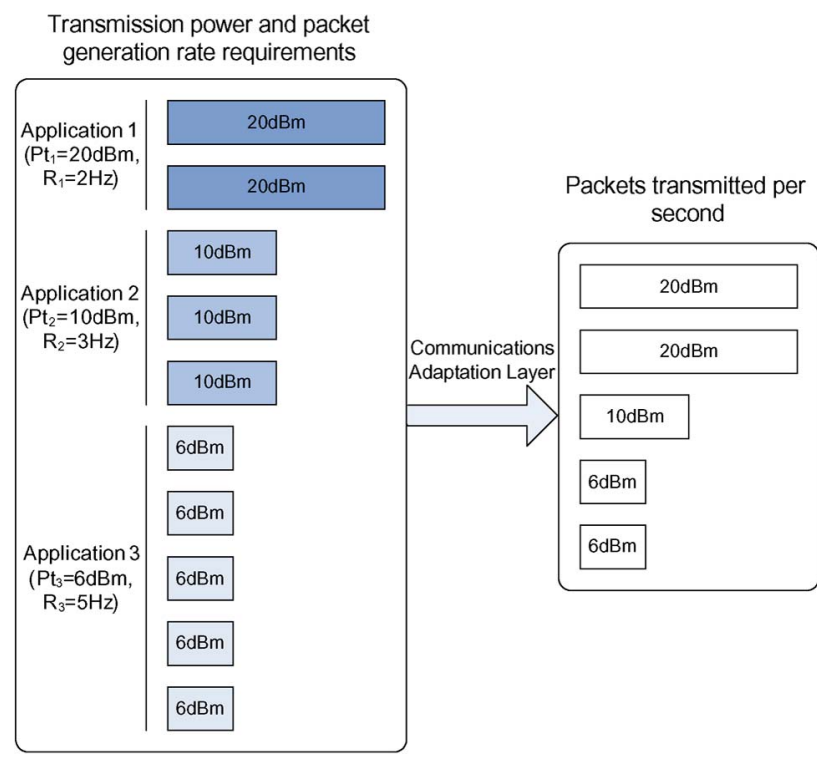

Fig. 14. Example of the communications adaptation layer to combine the different communication and applications requirements of various simultaneous applications.

requirements of the three applications in terms of packet generation rate. Two of these packets would need to be transmitted with at least $20 \mathrm{dBm}$ to satisfy application 1, three packets with at least $10 \mathrm{dBm}$ to satisfy application 2, and five packets with at least $6 \mathrm{dBm}$ to satisfy application 3.

Although this example clearly shows that the consideration of a CAL is certainly more efficient than treating each application separately, it also highlights the need for further research investigating optimization approaches to address multi-application scenarios. For example, considering the example shown in Fig. 14, the communications settings could be redefined if transmitting five packets with just $6 \mathrm{dBm}$ could directly satisfy the requirements of applications 1 and 2. This would reduce the channel load and increase the system capacity with respect to the solution discussed in the figure. In addition, the proposed CAL should be designed considering its interaction with the contribution from [45], which proposes to combine the information to be transmitted by different applications to reduce the channel load generated by each vehicle. This contribution focused on the payload of different application messages, but not on the configuration of the communication resources. The efficient combination of the required transmission parameters and the information to be transmitted by the different applications could then be part of the optimization of the proposed adaptation layer. The integration of all these multi-application considerations constitutes an interesting and open research field that should be addressed by the cooperative vehicular systems research community.

\section{CONCLUSION}

Congestion and awareness control techniques represent relevant building blocks in cooperative vehicular communications, since they are essential mechanisms to ensure the stable and reliable operation of communications system, while efficiently using the limited channel bandwidth. This paper has presented a unified view of the underlying control issues, and has also clarified the different perspectives that congestion control and awareness control proposals have taken in the past. Different approaches such as D-FPAV and OPRAM served as specific examples for congestion control and awareness control, respectively.

When drawing conclusions from the survey of options we have presented, one should differentiate between a first-stage deployment and full and wide deployment. To get a "first generation" of cooperative vehicular communications deployed, it might be helpful to assume a small set of different application classes. In this case, congestion control is well understood, and relatively simple methods like the reactive approaches based on distributed information about the currently used transmit parameters and channel congestion, provide a good performance versus complexity tradeoff somewhat similar to overprovisioning strategies in other networks. The design of awareness control policies could also be simplified and an individual application approach could be feasible. A full-scale deployment in large and multi-application scenarios might require the use of advanced congestion and awareness control policies. However, tackling joint congestion control and awareness control can be highly complex, in consideration of the different and possibly contradicting requirements of the vehicles involved. In this context, advances in information theory for local broadcast networks as well as application of operations research techniques, for example, game theory, might help as foundations for a theory of optimal communications setting in cooperative vehicular systems: an information theory for local broadcast networks could define what exactly is possible in these types of networks from a local broadcast capacity point of view, while the operations research related treatment could help in dealing with contradicting system and application requirements.

Finally, the issue of congestion control and awareness control depends heavily on allocated frequency bands, medium access control techniques, and available technology, and will definitely have to be revised with regulatory and technical advances.

\section{Acknowledgment}

J. Mittag would like to acknowledge the support of the European Telecommunications Standard Institute (ETSI) for the Specialist Task Force (STF) 395 on Configuration and validation of channel congestion control methods of ITS, as well as all members of this STF for many interesting discussions. 


\section{REFERENCES}

[1] IEEE P802.11p/D11.0, Draft Amendment for Wireless Access in Vehicular Environments (WAVE), Apr. 2010.

[2] ETSI TC ITS, Intelligent Transport Systems (ITS); European Profile Standard on the Physical and Medium Access Layer of 5 GHz ITSs, Draft ETSI ES 202663 V0.0.6, Oct. 2009.

[3] P. Santi, "Topology control in wireless ad hoc and sensor networks," ACM Comput. Surv., vol. 37, no. 2, pp. 164-194, 2005.

[4] L. Cheng, B. Henty, R. Cooper, D. Stancil, and F. Bai, "Multi-path propagation measurements for vehicular networks at $5.9 \mathrm{GHz}$," in Proc. Wireless Commun. Netw. Conf., Apr. 2008, pp. 1239-1244.

[5] I. N. L. Tan, W. Tang, K. Laberteaux, and A. Bahai, "Measurement and Analysis of Wireless Channel Impairments in DSRC Vehicular Communications," EECS Dept. Univ. California, Berkeley, Tech. Rep. UCB/EECS-2008-33. [Online]. Available: http://www.eecs.berkeley.edu/Pubs/ TechRpts/2008/EECS-2008-33.html

[6] Y. Zhang, I. L. Tan, C. Chun, K. Laberteaux, and A. Bahai, "A differential ofdm approach to coherence time mitigation in dsrc," in Proc. VANET'08: Proc. Fifth ACM Int. Workshop on Veh. Inter-NETworking, New York, 2008, pp. 1-6ACM.

[7] P. Alexander, D. Haley, and A. Grant, "Outdoor mobile broadband access with 802.11," IEEE Commun. Mag., vol. 45, no. 11, pp. 108-114, Nov. 2007.

[8] Federal Communications Commision. FCC 03-324. FCC Report and Order, Feb. 2004.

[9] D. Jiang, Q. Chen, and L. Delgrossi, "Optimal data rate selection for vehicle safety communications," in Proc. VANET'08: Proc. Fifth ACM Int. Workshop on Veh. Inter-NETworking, New York, USA, 2008, pp. 30-38ACM.

[10] T. R. Board, Highway Capacity Manual. Washington, DC: National Academy of Science, 2000.

[11] Q. Xu, R. Sengupta, and D. Jiang, "Design and analysis of highway safety communication protocol in $5.9 \mathrm{GHz}$ dedicated short range communication spectrum," in Proc. IEEE 57th Veh. Technol. Conf. (VTC-Spring), Jeju, Korea, Oct. 2004, pp. 2451-2455.

[12] H.-J. Reumerman, M. Roggero, and M. Ruffini, "The application-based clustering concept and requirements for intervehicle networks," IEEE Commun. Mag., vol. 42, no. 4, pp. 108-113, Apr. 2005.

[13] VSC, "Task 3 final report-Identify intelligent vehicle safety applications enabled by DSRC," in Public Document, Vehicle Safety Communications Project, Crash Avoidance Metrics Partnership, 2004.

[14] M. Torrent-Moreno, P. Santi, and H. Hartenstein, "Fair sharing of bandwidth in VANETs," in Proc. ACM Int. Workshop on Veh. Inter-Networking (VANET), Cologne, Germany, Sep. 2005, pp. 49-58.

[15] T. ElBatt, S. K. Goel, G. Holland, H. Krishnan, and J. Parikh, "Cooperative collision warning using dedicated short range wireless communications," in Proc. ACM Int. Workshop on Veh. Inter-Networking (VANET), Los Angeles, CA, Sep. 2006, pp. 1-9.

[16] S. Rezaei, R. Sengupta, H. Krishnan, X. Guan, and R. Bhatia, "Tracking the position of neighboring vehicles using wireless communications," Transport. Res. Part C: Emerg. Technol., vol. 18, no. 3, pp. 335-350, Jun. 2010.

[17] X. Yang, J. Liu, F. Zhao, and N. Vaidya "A vehicle-to-vehicle communication protocol for cooperative collision warning," in Proc. First Annu. Int. Conf. Mobile and Ubiquitous Syst., Netw. Services (MobiQuitous), Boston, USA, Aug. 2004, pp. 1-10.

[18] H. Xu and M. Barth, "A transmission-interval and power-level modulation methodology for optimizing inter-vehicle communications," in Proc. ACM Int. Workshop on Veh. Inter-Networking (VANET), Philadelphia, PA, Oct. 2004, pp. 97-98.

[19] M. Artimy, W. Robertson, and W. Phillips, "Assignment of dynamic transmission range based on estimation of vehicle density," in Proc. ACM Int. Workshop on Veh. Inter-Networking (VANET), Cologne, Germany, Sep. 2005, pp. 40-48.

[20] M. Torrent-Moreno, J. Mittag, P. Santi, and H. Hartenstein, "Vehicle-to-vehicle communication: Fair transmit power control for safety-critical information," IEEE Trans. Veh. Technol., vol. 58, pp. 3684-3703, Sep. 2009.

[21] C. Khorakhun, H. Busche, and H. Rohling, "Congestion control for VANETs based on power or rate adaptation," in Proc. Int Workshop on Intelligent Transportation (WIT), Hamburg, Germany, Mar. 2008.

[22] R. Baldessaro, D. Scanferla, L. Le, W. Zhang, and A. Festag, "Joining forces for VANETs: A combined transmit power and rate control algorithm," in Proc. Int. Workshop on Intelligent Transport. (WIT), Hamburg, Germany, Mar. 2010.

[23] C.-L. Huang, Y. Fallah, R. Sengupta, and H. Krishnan, "Adaptive intervehicle communication control for cooperative safety systems," IEEE Network, vol. 24, no. 1, pp. 6-13, Jan. 2010.

[24] J. Mittag, F. Schmidt-Eisenlohr, M. Killat J. Härri, and H. Hartenstein, "Analysis and design of effective and low-overhead transmission power control for VANETs," in Proc. ACM Int. Workshop on Veh. Inter-Networking (VANET), San Francisco, CA, Sep. 2008, pp. 39-48.

[25] W. Zhang, A. Festag, R. Baldessari, and L. Le, "Congestion control for safety messages in VANETs: Concepts and framework," in Proc. Int. Conf. ITS Telecommun. (ITST), Pucket, Thailand, Oct. 2008, pp. 199-203.

[26] VSC Consortium, "Vehicle safety communications project task 3-Final report: Identify intelligent vehicle safety applications enabled by DSRC," US DOT HS 809 859, Mar. 2005.

[27] ETSI TC ITS, "Intelligent transport systems (ITS); Vehicular communications; Basic set of applications; Definitions," ETSI TR 102638 V1.1.1, Jun. 2009.

[28] Q. Xu, T. Mak, J. Ko, and R. Sengupta, "Vehicle-to-vehicle safety messaging in DSRC," in Proc. ACM Int. Workshop on Veh. Inter-Networking (VANET), Philadelphia, PA Oct. 2004, pp. 19-28.

[29] F. Bai and H. Krishnan, "Reliability analysis of DSRC wireless communication for vehicle safety applications," in Proc. IEEE Intelligent Transport. Syst. Conf. (ITSC), Toronto, ON, Canada, Sep. 2006, pp. 355-362.

[30] J. Gozalvez and M. Sepulcre, "Opportunistic technique for efficient wireless vehicular communications," IEEE Veh. Technol. Mag., vol. 2, no. 4, pp. 33-39, Dec. 2007.
[31] T. Osafune, L. Lin, and M. Lenardi, "Multi-hop vehicular broadcast (MHVB)," in Proc. Int. Conf. ITS Telecommun. (ITST), Chengdu, China, Jun. 2006, pp. 757-760.

[32] L. Yang, J. Guo, and Y. Wu, "Piggyback cooperative repetition for reliable broadcasting of safety messages in VANETs," in Proc. IEEE Consumer Commun. Netw. Conf. (CCNC), Las Vegas, NV, Jan. 2009 pp. 1165-1169.

[33] J. Mittag, F. Thomas, J. Härri, and H. Hartenstein, "A comparison of single- and multi-hop beaconing in VANETs," in Proc. ACM Int. Workshop on Veh. Inter-Networking (VANET), Beijing, China, Sep. 2009, pp. 69-78.

[34] M. Ruffini and H.-J. Reumerman, "Power-rare adaptation in high-mobility distributed ad-hoc wireless networks," in Proc. IEEE Veh. Technol. Conf. (VTC-Spring), Stockholm, Sweden, May 2005, vol. 4, pp. 2299-2303.

[35] X. Guan, R. Sengupta, H. Krishnan, and F. Bai, "A feedback-based power control algorithm design for VANET," in Proc. Mobile Netw. Veh. Environ. Workshop (MOVE), Anchorage, AK, May 2007, pp. 67-72.

[36] Q. Xu, T. Mak, J. Ko, and R. Sengupta, "Medium access control protocol design for vehicle-vehicle safety messages," IEEE Trans. Veh. Technol., vol. 56, no. 2, pp. 499-518, Mar. 2007.

[37] R. Schmidt, T. Leinmuller, E. Schoch, F. Kargl, and G. Schafer, "Exploration of adaptive beaconing for efficient intervehicle safety communication," IEEE Network, vol. 24, no. 1, pp. 14-19, Jan. 2010.

[38] WINNER Consortium, "WINNER II channel models," WINNER European Research project Public Deliverable D1.1.2., Sep. 2007.

[39] M. Sepulcre and J. Gozalvez, "Wireless vehicular adaptive radio resource management policies in congested channels," in Proc. IEEE Int. Symp. Wireless Commun. Syst. (ISWCS), Trondheim, Norway, Oct. 2007, pp. 380-384.

[40] M. Sepulcre and J. Gozalvez, "Adaptive wireless vehicular communication techniques under correlated radio channels," in Proc. IEEE Veh. Technol. Conf. (VTC-Spring), Barcelona, Spain, Apr. 2009, pp. 1-5.

[41] J. Gozalvez and M. Sepulcre, "Channel efficiency of adaptive transmission techniques for wireless vehicular communications," in Proc. Int. World Congr. on ITS, New York, Nov. 2008.

[42] V. Taliwal, D. Jiang, H. Mangold, C. Chen, and R. Sengupta, "Empirical determination of channel characteristics for DSRC vehicle-to-vehicle communication," in Proc. ACM Int. Workshop on Veh. Inter-Networking (VANET), Philadelphia, PA, Oct. 2004, p. 88-88.

[43] T. Kosch, C. Adler, S. Eichler, C. Schroth, and M. Strassberger, "The scalability problem of vehicular ad hoc networks and how to solve it," IEEE Wireless Commun., vol. 13, no. 5, pp. 22-28, Oct. 2006.

[44] Geonet Consortium, "Final GeoNet specification," GeoNet European Research project Public Deliverable D2.2, Jan. 2010.

[45] C. Robinson, D. Caveney, L. Caminiti, G. Baliga, K. Laberteaux, and P. Kumar, "Efficient message composition and coding for cooperative vehicular safety applications," IEEE Trans. Veh. Technol., vol. 56, no. 6, pp. 3244-3255, Nov. 2007. 


\section{ABOUT THE AUTHORS}

Miguel Sepulcre received the degree in telecommunications engineering in 2004 and the Ph.D. degree in communications technologies in 2010, both from the University Miguel Hernandez of Elche (UMH), Spain.

In 2004, he spent six months at the European Space Agency (ESA), Noordwijk, The Netherlands, working on the communications physical layer of Earth exploration satellites. In 2005, he joined the University Miguel Hernandez of Elche as a Net-

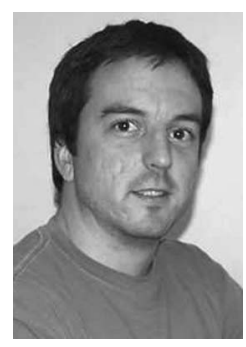
works Manager and Teaching Assistant. In March 2006, he received a Ph.D. fellowship from the Valencian regional government and joined the Uwicore research laboratory. In 2009, he spent three months at the Karlsruhe Institute of Technology (KIT), Karlsruhe, Germany, working on cooperative vehicular communications. He is currently a Research Fellow at the Uwicore Research Laboratory of $\mathrm{UMH}$, working on cooperative vehicular communications, radio resource management, and modeling and simulation of wireless communications systems.

Jens Mittag received the Diploma in computer science from the University of Karlsruhe, Germany, in 2008. He is currently working towards the Ph.D. degree within the Decentralized Systems and Network Services Research Group at the Karlsruhe Institute of Technology (KIT), Germany.

His research interests include vehicular mobile networks, accurate simulation environments and the modeling of the wireless lower physical layers.

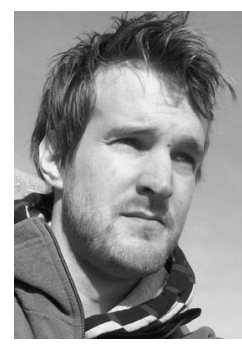
He participated in the NOW: Network on Wheels project (2004-2008) and contributed to the standardization of intelligent transportation systems (ITS) in Europe as a member of the European Telecommunications Standards Institute (ETSI) specialist task force on Configuration and Validation of Channel Congestion Control Methods of ITS.

Paolo Santi (Member, IEEE) received the Laura degree (summa cum laude) and the Ph.D. degree in computer science from the University of Pisa, Italy, in 1994 and 2000, respectively.

He has been Researcher at the Istituto di Informatica e Telematica-CNR, Pisa, since 2001, and Senior Researcher since 2009. During his career, he visited Georgia Institute of Technology, Atlanta, in 2001 and Carnegie Mellon University, Pittsburgh, PA, in 2003. His research interests

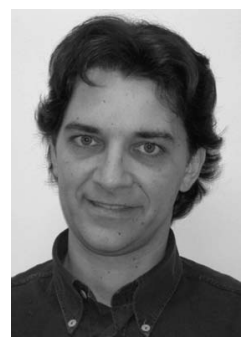
include fault-tolerant computing in multiprocessor systems (during his Ph.D. studies), and more recently, the investigation of fundamental properties of wireless multihop networks such as connectivity, topology control, lifetime, capacity, mobility modeling, and cooperation issues. He has contributed to more than 50 papers and a book in the field of wireless ad hoc and sensor networking.

Dr. Santi has been General Co-Chair of ACM VANET 2007/2008, Technical Program Co-Chair of IEEE WiMesh 2009, and he is involved in the organizational and technical program committee of several conferences in the field. He is an Associate Editor for IEEE TRANSACTIONS ON Mobile Computing and ieEe transactions on Parallel and Distributed SYSTEMS. He is a member of IEEE Computer Society and a Senior member of ACM and SIGMOBILE.
Hannes Hartenstein (Member, IEEE) received the Diploma in mathematics and the Ph.D. degree in computer science from Albert-LudwigsUniversität, Freiburg, Germany, in 1995 and 1998, respectively.

He is a Full Professor for decentralized systems and network services at the Karlsruhe Institute of Technology (KIT), Germany, and Executive Director of the KIT Steinbuch Centre for Computing. His research interests include mobile networks, vir-

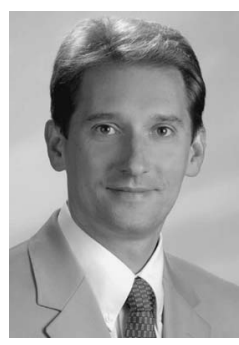
tual networks and IT management. Prior to joining the University of Karlsruhe, he was a Senior Research Staff Member with NEC Europe.

Dr. Hartenstein was involved in the FleetNet-Internet on the Road (2000-2003) and NOW: Network on Wheels (2004-2008) projects, partly funded by the German Ministry of Education and Research (BMBF), he actively participated in the EU FP7 project PRE-DRIVE-C2X (2008-2010), and is now contributing to the follow-on project called DRIVE-C2X. He has been TPC Co-Chair and General Chair of various highly selective ACM and IEEE international workshops and symposia on vehicular communications.

Javier Gozalvez (Senior Member, IEEE) received the degree in electronics engineering from the French Engineering School ENSEIRB and the Ph. D. degree in mobile communications from the University of Strathclyde, Glasgow, U.K.

Since October 2002, he has been with the University Miguel Hernandez of Elche, Spain, where he is currently an Associate Professor and Director of the Uwicore Research Laboratory. At Uwicore, he is leading research activities in the

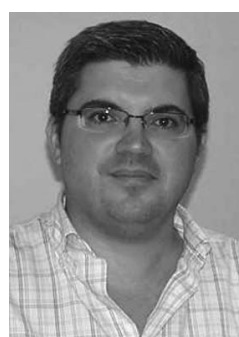
areas of wireless vehicular communications, radio resource management, heterogeneous wireless systems, and wireless system design and optimization.

Dr. Gozalvez currently serves as Mobile Radio Senior Editor of IEEE Vehicular Technology Magazine, and previously served as AE of IEEE COMmUnication LetTeRs. He was TPC Co-Chair of the 2009 IEEE VTC Spring, and General Co-Chair of the 3rd International Symposium on Wireless Communications Systems (ISWCS'2006). He is also the founder and General Co-Chair of the IEEE International Symposium on Wireless Vehicular Communications (WiVeC) in its 2007, 2008, and 2010 editions. $\mathrm{He}$ has recently been elected to the Board of Governors of the IEEE Vehicular Technology Society (2011-2014). 